\title{
THE EFFECT OF STRAIN RATE ON DIFFUSION FLAMES*
}

\author{
G. F. CARRIER $\dagger$, F. E. FENDELL $\ddagger$, AND F. E. MARBLEף
}

\begin{abstract}
Several steady state and time-dependent solutions to the compressible conservation laws describing direct one-step near-equilibrium irreversible exothermic burning of initially unmixed gaseous reactants, with Lewis-Semenov number unity, are presented. The quantitative investigation first establishes the Burke-Schumann thin-flame solution using the Shvab-Zeldovich formulation. Real flames do not have the indefinitely thin reaction zone associated with the Burke-Schumann solution. Singular perturbation analysis is used to provide a modification of the thin-flame solution which includes a more realistic reaction zone of small but finite thickness. The particular geometry emphasized is the unbounded counterflow such that there exists a spatially constant rate of strain along the flame. While the solutions for diffusion flames under a finite tangential strain rate may be of interest in and of themselves for laminar flow, the problems are motivated by the authors' belief that they are pertinent to the study of diffusion-flame burning in transitional and turbulent shear flows.
\end{abstract}

1. Introduction. Experiments (e.g., Brown and Roshko [1]) concerning the initial mixing region between two parallel streams suggest that the interface between the two streams remains relatively intact for a significant distance from the point of initiation. Dynamically this implies that the thickness of the laminar mixing zone is small in comparison with the wavelength of disturbances that grow most rapidly. The interface, although severely contorted and strained, remains visually recognizable.

When the process involves two gases that react chemically upon mixing, one may conjecture that the resulting diffusion flame behaves analogously to the interface between the two streams. That is, it is severely distorted and strained but retains its identity. More specifically, the thickness of the diffusion flame is small in comparison with the predominant disturbance wavelength so that the flame is aware of only the local gas rotation and strain rates. But since the rotations themselves are irrelevant, the diffusion flame structure is affected only by the strain rate in its own plane.

Then, if one inquires into the "turbulent reaction rate," it is clearly augmented over the laminar reaction rate by (i) the greatly increased arc length of flame front and (ii) the local increase in fuel reacted per unit length caused by the linear strain rate.

The last statement, that the local fuel consumption per unit length of flame increases with strain rate, is suggested because the inflow of gas toward the reaction front caused by the strain rate augments the diffusion process in transporting

* Received by the editors March 19, 1974. This work was supported in part by: Project SQUID, supported by the Office of Naval Research, Department of the Navy, under Contract N00014-67-A0226-0005, NR-098-038 (F.E.F.); Office of Naval Research Contract N00014-67-A-0298-0033 and National Science Foundation Contract NSF-GP-34723 (G.F.C.). Presented by invitation at an International Symposium on Modern Developments in Fluid Dynamics in Honor of the 70th Birthday of Sydney Goldstein held at Haifa, Israel, December 16-23, 1973.

† Division of Engineering and Applied Physics, Harvard University, Cambridge, Massachusetts 02138 .

¥ Fluid Mechanics Laboratory, TRW Systems, Redondo Beach, California 90278.

I Division of Engineering and Applied Science, California Institute of Technology, Pasadena, California 91109. 
reactants toward the flame. But it must be remembered that the chemical reaction rate is finite so that for very high strain rates the rapid transport of cool material toward the flame may quench the reaction and actually reduce the local fuel consumption rate.

Now as the distortion of the interface continues, one finds regions where the straining process brings neighboring parallel flame fronts closer to one another until they are no longer thermochemically independent. The situation is characterized by a thin strip of fuel bounded by two regions of oxidizer with diffusion flames at the interfaces. In time, these two flames move together, consume the intervening fuel, and remove the two lengths of flame from the field. The picture may equally well be put in terms of a spherical or cylindrical fuel mass surrounded by flame, since the essential conclusion is the same.

This is the flame shortening mechanism that eventually counteracts the extension caused by straining. It seems probable that, in the early stages of turbulent combustion of unmixed reactants, the competition between (i) straining and (ii) mutual annihilation of diffusion flames establishes the level of fuel consumption per unit volume. It is for this reason that these two aspects of laminar flame theory have been chosen for examination.

2. Formulation. We are concerned, generally, with the influence of the distortion associated with a time-dependent motion on the changes in temperature and species populations which occur in the moving reacting fluid. In particular, in this paper, we investigate local features of such configurations in which, as in Fig. 1, the Cartesian coordinate system moves with one particle in the region of interest
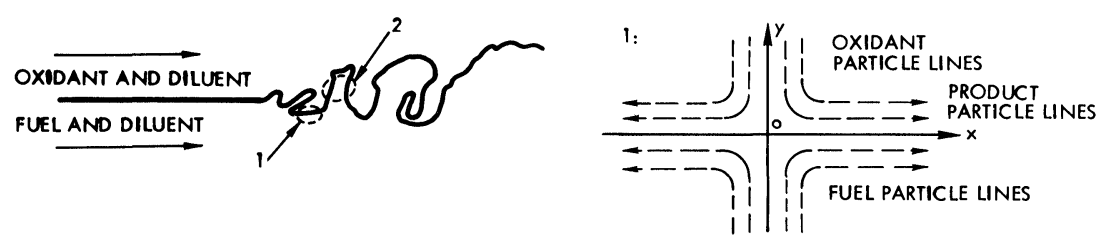

FIG. 1. Left, a schematic of the highly convoluted interface extending downstream from the splitter plate for a two-dimensional mixing zone with initially unmixed gaseous reactants. This interface, related to the large-scale structure recently noted in free shear layers, is conjectured to separate fuel and oxidant; that is, there is little interdiffusion across the interface. A magnification of the local flow seen by an observer located on the rapidly oscillating interface at region 1 is shown to the right; if burning occurs in the vicinity of the interface, this is a single-flame region. Region 2 typifies a thin strip of fuel penetrating into oxidant such that locally a double-flame region is formed.

and rotates at such a rate that, locally, the flame front (or fronts) remain parallel to (or coincident with) the $x$-axis. In that coordinate system we adopt a velocity field $\hat{x} u(x, y, t)+\hat{y} v(x, y, t)$ such that

$$
u_{x}(x, y, t)=\varepsilon(t)
$$

We confine our attention to problems in which the dependence on $x$ of all quantities of interest is so small that it can be ignored. 
As is appropriate in deflagration phenomena, we also limit our studies to configurations in which the pressure gradient plays a negligible role.

With conventional choice of variable, as listed below, and with ${ }^{1} \rho c_{p} D / k=1$, the consumption of reactants and the evolution of heat are described by the equations

$$
\begin{aligned}
& L(h)=B Y^{v_{0}} F^{v_{F}} \exp \left(-\frac{\theta}{h+\alpha}\right), \\
& L(Y+h)=L(F+h)=0,
\end{aligned}
$$

where

$$
L=\frac{\partial}{\partial t}-\varepsilon(t) \xi \frac{\partial}{\partial \xi}-\frac{\partial}{\partial \xi}\left[\left(\frac{\rho}{\rho_{\infty}}\right)^{2} D \frac{\partial}{\partial \xi}\right]
$$

In this formulation, the reaction has been modeled as a one-step chemical process. Ordinarily, if the reaction really is a one-step process, each of the stoichiometric coefficients $v_{0}$ and $v_{F}$ will be unity, but when the right-hand side of (2.1) is merely a one-step model of a more complicated process, $v_{0}$ and $v_{F}$ can take on other positive values (Williams [17]).

In the foregoing equations $t$ is the time, $\xi=\int_{0}^{y}\left[\rho\left(x, y^{\prime}, t\right) / \rho_{\infty}\right] d y^{\prime}, \rho_{\infty}=\rho(x$, $\infty, t), Y(\xi, t)$ and $F(\xi, t)$ are the (stoichiometrically adjusted) mass fractions of oxidant and fuel, the dimensionless enthalpy is defined by $h=c_{p}\left(T-T_{0}\right) / \Delta H$, where $T$ is the temperature, $T_{0}=298.16^{\circ} \mathrm{K}$, and $\Delta H$ is the specific heat of reaction of the reactants at $T_{0}$ and 1 atmosphere pressure. In the exponential function of (2.1), $\alpha=c_{p} T_{0} / \Delta H$, and $\theta$ is the activation temperature of Arrhenius kinetics [16]. ${ }^{2}$

TABLE 1

Parameter values for representative rapid second order chemical reactions. The frequency factors given in [9] are taken to increase linearly with temperature. The quantity $\Delta H$ is the heat of reaction per unit mass of (stoichiometrically weighted) reactants. Atmospheric pressure has been adopted in characterizing the preexponential factor $B$ of the law of mass action; $R^{0}$ is the universal gas constant. The value of the strain rate adopted is $\varepsilon_{0}=10^{6} \mathrm{sec}^{-1}$; this is the maximum instantaneous value likely to be achieved in a subsonic shear layer.*

\begin{tabular}{lccccccc} 
& $\frac{B_{f}}{T}\left[\frac{\mathrm{cm}^{3}}{\text { mole-sec- }{ }^{-} \mathrm{K}}\right]$ & $\theta_{f}\left[{ }^{\circ} \mathrm{K}\right]$ & $\Delta H\left[\frac{\mathrm{cal}}{\mathrm{gm}}\right] \alpha \equiv \frac{c_{p} T_{0}}{\Delta H} \theta \equiv \frac{c_{p} \theta_{f}}{\Delta H}$ & $B \equiv \frac{B_{f} p_{\infty}\left[\mathrm{sec}^{-1}\right]}{R^{0}}$ & $D_{1} \approx \frac{B}{\varepsilon_{0}}$ \\
\hline $\mathrm{OH}+\mathrm{CO} \rightarrow \mathrm{CO}_{2}+\mathrm{H}$ & $71 \times 10^{13}$ & 3875 & 569 & .17 & 2.42 & $8.7 \times 10^{12}$ & $9 \times 10^{4}$ \\
$\mathrm{OH}+\mathrm{CH}_{4} \rightarrow \mathrm{CH}_{3}+\mathrm{H}_{2} \mathrm{O}$ & $50 \times 10^{13}$ & 4980 & 460 & .29 & 5.25 & $6.1 \times 10^{12}$ & $6 \times 10^{6}$ \\
$\mathrm{H}+\mathrm{Br}_{2} \rightarrow \mathrm{HBr}+\mathrm{Br}$ & $45 \times 10^{13}$ & 1450 & 381 & .07 & 0.38 & $5.5 \times 10^{12}$ & $6 \times 10^{6}$ \\
\hline
\end{tabular}

\footnotetext{
${ }^{1} \rho$ is the density of the fluid, $c_{p}$ is its (constant) specific heat, $D$ is the mass diffusivity, and $k$ is the thermal conductivity.

${ }^{2}$ Implicit in the formulation leading to (2.1) is the choice, for tractability, of $\tilde{\alpha}=\left(v_{0}+v_{F}-1\right)$ in the Arrhenius rate expression $B_{f} T^{\tilde{\alpha}} \exp [-\theta /(h+\alpha)]$. The frequency factor $B_{f}$ is a factor in the preexponential constant $B$.

* The authors are indebted to Prof. Richard Kaplan, Department of Aerospace Engineering, University of Southern California, for these data.
} 
The preexponential (constant) factor $B$ has dimensions $t^{-1}$ and varies greatly from one set of reactants to another. Table 1 indicates the values of these parameters for a few reactant pairs. An important characterizing feature of these numbers is associated with the fact that the factor $Z=B \exp \{-\theta /(h+\alpha)\}$ is very large when measured in $\mathrm{sec}^{-1}$.

A detailed account of the development of the foregoing (so-called) ShvabZeldovich formulation of the problem under a Howarth transformation can be found in Williams [16].

In order to render (2.1) and (2.2) dimensionless, we must refer each quantity with units $t$ to some naturally occurring reference time, and refer $\xi$ to a reference length, $l$. In this situation the externally imposed time of interest is $\varepsilon^{-1}$ evaluated at a time of particular interest in the phenomenon. For each problem we consider we can best identify such a scale later, so, for now, we merely denote it by $\varepsilon_{0}^{-1}$. We then define the length scale to be

$$
l=\left(D_{\infty} / \varepsilon_{0}\right)^{1 / 2},
$$

and take $\rho^{2} D=\rho_{\infty}^{2} D_{\infty}$, an excellent approximation for a nearly isobaric flow.

We continue to use the symbol $t$ as the dimensionless time, $\varepsilon$ as the nondimensional strain rate, and $\xi$ as a nondimensional coordinate, but we replace $B / \varepsilon_{0}$ by $D_{1}$ in conformity with conventional notations for this "first Damköhler number." (Studies are carried out here for $D_{1}$ large, i.e., for flows near chemical equilibrium.)

Thus, the equations we work with are

$$
\begin{aligned}
& L(h)=D_{1} Y^{v_{0}} F^{v_{F}} \exp \left\{-\frac{\theta}{h+\alpha}\right\}, \\
& L(Y+h)=L(F+h)=0,
\end{aligned}
$$

where

$$
L=\frac{\partial}{\partial t}-\varepsilon(t) \xi \frac{\partial}{\partial \xi}-\frac{\partial^{2}}{\partial \xi^{2}} .
$$

In the problems of interest here, each set of initial conditions will be chosen to initiate, in as simply described a manner as is possible, both single flame configurations and double flame configurations. These initial conditions turn out to be ${ }^{3}$

$$
\begin{aligned}
& \xi>m_{0}: Y=Y_{\infty}, F=0, h=h_{\infty} ; \\
& \xi<m_{0}: Y=0, F=F_{-\infty}, h=h_{-\infty}, \\
& \xi>\left|m_{0}\right|: Y=Y_{\infty}, F=0, h=h_{\infty} ; \\
& \xi<\left|m_{0}\right|: Y=0, F=F_{i}, h=h_{i} .
\end{aligned}
$$

The boundary conditions which are pertinent to each of these studies merely require that as $\xi \rightarrow \pm \infty$ the values of $Y, F$, and $h$ never depart from their initial values.

3. The steady state solution. It is well known that if $\varepsilon=$ const. and if we seek only the steady state configuration $Y(\xi), F(\xi), h(\xi),(2.4)$ and (2.5) admit solutions in which each of $Y+h, F+h, Y-F$ is a linear combination of 1 and $\operatorname{erf} \zeta$, where $\zeta=(\varepsilon / 2)^{1 / 2} \xi$.

\footnotetext{
${ }^{3}$ The quantities with subscripts $\infty,-\infty$, or $i$ in (2.7) are constants; methods for treating more general initial conditions are discussed below.
} 
Furthermore, in the limit $D_{1} \rightarrow \infty$, and with the boundary conditions

$$
\begin{array}{cc}
Y(-\infty)=0, & Y(\infty)=Y_{\infty} ; \quad F(-\infty)=F_{\infty}, \quad F(\infty)=0 ; \\
& h(\infty)=h_{\infty}, \quad h(-\infty)=h_{-\infty},
\end{array}
$$

$Y, F$, and $h$ are given by

$$
\begin{array}{ll}
Y+h=c_{1} \operatorname{erf} \zeta+c_{2}, & F+h=c_{3} \operatorname{erf} \zeta+c_{4}, \\
2 c_{1}=Y_{\infty}+h_{\infty}-h_{-\infty}, & 2 c_{2}=Y_{\infty}+h_{\infty}+h_{-\infty}, \\
2 c_{3}=h_{\infty}-h_{-\infty}-F_{\infty}, & 2 c_{4}=h_{\infty}+h_{-\infty}+F_{\infty} .
\end{array}
$$

This result is depicted in Fig. 2. It is noteworthy that $Y F=0$ at each $\zeta$; that the

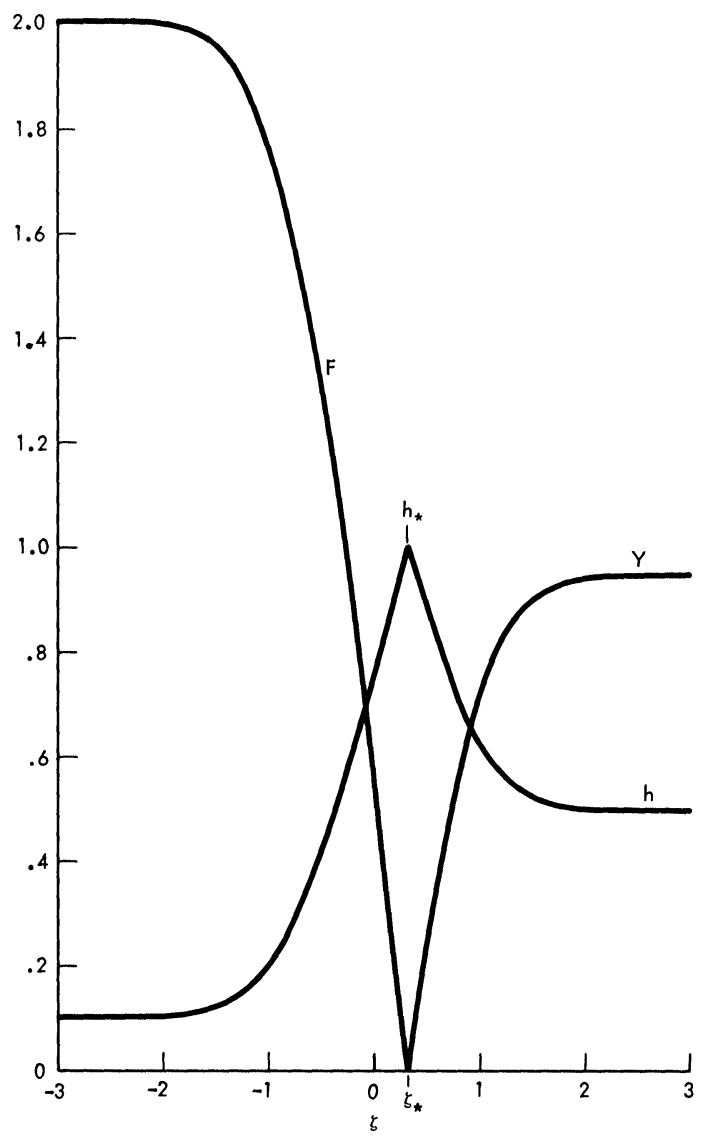

FIG. 2. The thin-flame (Burke-Schumann) solution for a one-step irreversible reaction in equilibrium for a counterflow. The stoichiometrically adjusted mass fractions for fuel and oxidant $F$ and $Y$ and the dimensionless sensible enthalpy $h$ are plotted against the spatial coordinate $\zeta$. For $Y_{\infty}=0.95$, $F_{\infty}=2.0, h_{\infty}=0.5$, and $h_{-\infty}=0.1$, the adiabatic flame enthalpy $h_{*} \doteq 1.015$, and the position of the thin flame $\zeta_{*}=0.327$. 
indefinitely thin flame lies at $\zeta=\zeta_{*}$ (so $Y=0$ for $\zeta \geqq \zeta_{*}, F=0$ for $\zeta \leqq \zeta_{*}$ ) where

$$
\operatorname{erf} \zeta_{*}=\frac{F_{\infty}-Y_{\infty}}{F_{\infty}+Y_{\infty}}
$$

Further, the so-called adiabatic flame temperature

$$
h_{*} \equiv h\left(\zeta_{*}\right)=\frac{F_{\infty} Y_{\infty}+h_{\infty} F_{\infty}+h_{-\infty} Y_{\infty}}{Y_{\infty}+F_{\infty}}
$$

this is the highest temperature achievable in the flow. The flux of reactants into the flame is given by

$$
\text { flux }=\left|\frac{d Y\left(\xi_{*+}\right)}{d \xi}\right|=\left|\frac{d F\left(\xi_{*-}\right)}{d \xi}\right|=\left|\frac{F_{\infty}+Y_{\infty}}{(2 \pi)^{1 / 2}} \varepsilon^{1 / 2} \exp \left(-\zeta_{*}^{2}\right)\right| .
$$

Since, from (3.4), $\zeta_{*}$ is a function of $F_{\infty}$ and $Y_{\infty}$ only, (3.6) indicates that the reaction consumption increases as $\varepsilon^{1 / 2}$. However, increasing the strain rate $\varepsilon$ also serves to decrease $D_{1}$, so (3.6), based on $D_{1} \rightarrow \infty$, eventually fails to hold.

When $D_{1}$ is large but finite, it is known that (3.1)-(3.3) subject to $Y F=0$ provide an excellent approximation to $Y, F$, and $h$ in all but a small part of the configuration. In that region, which includes $\xi=\xi_{*}$, the correction to each of $h, Y$, and $F$ is of order $\left(D_{1}\right)^{-1 /\left(1+v_{0}+v_{F}\right)}$ and the domain on which this correction is needed has a width of order $\left(D_{1}\right)^{-1 /\left(1+v_{0}+v_{F}\right)}$. Appendix A provides that analysis and documentation of those parts which go beyond what is already in the literature. Particularly notable in this analysis is the lack of importance, to lowest order of approximation, of the contributions of the convective term in either the corrective enthalpy balance or the corrective population balance.

4. Time-dependent configurations. One can use the Fourier transform in $\xi$ to initiate the construction of solutions of (2.4) and (2.5), but one quickly arrives at a point where it is evident that there are solutions analogous to those of the steady state equations. It is a simple matter, in fact, to verify that each of $Y-F$, $Y+h$, and $Y+F$ can be of the form $u$, where

$$
\begin{aligned}
& u=A+B \operatorname{erf}\left[\frac{\xi-m(t)}{r(t)}\right] \\
& r^{2}(t)=r_{0}^{2} \exp [-2 v(t)]+4 \exp [-2 v(t)] \int_{0}^{t} \exp \left[2 v\left(t^{\prime}\right)\right] d t^{\prime} \\
& m(t)=m_{0} \exp [-v(t)]
\end{aligned}
$$

and

$$
v(t)=\int_{0}^{t} \varepsilon\left(t^{\prime}\right) d t^{\prime}
$$

Furthermore, the solution $u(\xi, t)$ just given in (4.1) can be extended (sometimes usefully) to the form

$$
u=A+\sum_{j} B_{j} \operatorname{erf}\left[\frac{\xi-m_{j}(t)}{r_{j}(t)}\right]
$$

where each $m_{j}$ and $r_{j}$ is given by the obvious extension of (4.2) or (4.3). 
Actually, the transform method, in principle, can deal with much more general initial conditions than those which could accompany (4.5), but our purposes here are not aided by such generality. In fact, our purposes are best served by postulating initial conditions of a type, for example, where $m(0)=m_{0}$ and $r(0) \rightarrow 0$ in (4.1).

For a single thin flame $\left(D_{1} \rightarrow \infty\right)$ where, initially, the oxidant occupies $\xi>m_{0}$ with (stoichiometrically adjusted) mass fraction $Y_{\infty}$, the fuel occupies $\xi<m_{0}$ with mass fraction $F_{\infty}$, and the enthalpy $h(\xi, 0)$ is $h_{\infty}$ for $\xi>m_{0}$ and $h_{-\infty}$ for $\xi<m_{0}$ [as specified in (2.7a)], the subsequent configuration is described by

$$
Y+h=c_{1} \operatorname{erf}\left[\frac{\xi-m(t)}{r(t)}\right]+c_{2}, \quad F+h=c_{3} \operatorname{erf}\left[\frac{\xi-m(t)}{r(t)}\right]+c_{4}
$$

where $c_{1}, c_{2}, c_{3}, c_{4}$ were given in (3.1), (3.2). The position of the thin flame $\xi_{*}(t)$ is given by the condition $F=Y=0$ :

$$
Q\left[\xi_{*}(t), t\right] \equiv\left(c_{1}-c_{3}\right) \operatorname{erf}\left[\frac{\xi_{*}(t)-m(t)}{r(t)}\right]+\left(c_{2}-c_{4}\right)=0 ;
$$

a solution to this transcendental equation for $\xi^{*}(t)$ may perhaps be most readily found by integrating

$$
\frac{d \xi_{*}}{d t}=-\frac{(\partial Q / \partial t)_{\xi_{*}}}{(\partial Q / \partial \xi)_{t}}=\frac{d m}{d t}+\left[\xi_{*}(t)-m(t)\right] \frac{d[\ln r(t)]}{d t}
$$

integration of (4.8), with $\xi_{*}(t=0)=\xi_{0}$, gives

$$
\xi_{*}(t)=m(t)+\left[r(t) / r_{0}\right]\left[\xi_{0}-m_{0}\right] .
$$

For the case of interest, $r_{0} \rightarrow 0, \xi_{*}(t)=m(t)$ for all $t \geqq 0$. Accordingly for $\xi \geqq m(t)$,

$$
\begin{gathered}
Y=\left(c_{1}-c_{3}\right) \operatorname{erf}\left[\frac{\xi-m(t)}{r(t)}\right]+\left(c_{2}-c_{4}\right), \quad F=0, \\
h=c_{3} \operatorname{erf}\left[\frac{\xi-m(t)}{r(t)}\right]+c_{4}
\end{gathered}
$$

for $\xi \leqq m(t)$

$$
\begin{gathered}
Y=0, \quad F=\left(c_{3}-c_{1}\right) \operatorname{erf}\left[\frac{\xi-m(t)}{r(t)}\right]+\left(c_{4}-c_{2}\right), \\
h=c_{1} \operatorname{erf}\left[\frac{\xi-m(t)}{r(t)}\right]+c_{2} .
\end{gathered}
$$

At the thin flame $\xi=\xi_{*}(t)=m(t), h=h_{*}$, the adiabatic flame temperature given in (3.5), at all time $t$. Numerical results for typical cases in which $r_{0}$ is small but finite are given in Figs. 3 and $4 .^{4}$

${ }^{4}$ From (4.10), with (3.1) and (3.2), the rate of reactant consumption at the thin flame, nondimensionalized against $\rho_{\infty}\left(D_{\infty} \varepsilon_{0}\right)^{1 / 2}$, is

$$
\left|\frac{\partial Y\left(\xi=\xi_{*+}, t\right)}{\partial \xi}\right|=\left|\frac{\partial F\left(\xi=\xi_{*-}, t\right)}{\partial \xi}\right|=\frac{Y_{\infty}+F_{\infty}}{\pi^{1 / 2} r(t)} \exp \left\{-\left[\frac{\xi_{*}(t)-m(t)}{r(t)}\right]\right\},
$$


Once again the analysis of the corrections which are needed when $D_{1}$ is large but finite is relegated to an Appendix B. That analysis again serves to confirm that the thin flame picture is an excellent approximation to the true picture when $D_{1} \gg 1$.

In the Introduction, the protruding of a narrow strip of one reactant into the bulk of the other, owing to deep convolutions in the interface between them, was discussed. Such a strip could be pinched off to form a globule of one reactant entirely surrounded by the other reactant. If the globule is modeled as a gaseous sphere of fuel (say) with an initial spatially uniform (stoichiometrically adjusted) mass fraction $F_{i}$, and if this fuel sphere is taken to be immersed in an unbounded oxidant atmosphere characterized by $Y_{\infty}$ (this level being maintained in the ambient), then for incompressible constant-property conditions in the absence of any convection, Rosner [14], [15], [2] has noted that the thin flame for $D_{1} \rightarrow \infty$ first expands, then contracts if $F_{i}>Y_{\infty}$. Rosner found that the fuel is fully reacted at $\tau_{f} \equiv t_{f} /\left(a^{2} / D_{\infty}\right)$, where $a$ is the initial sphere radius and $t$ is dimensional time given by

$$
\operatorname{erf}\left(\frac{1}{2 \tau_{f}^{1 / 2}}\right)-\frac{\exp \left(-1 / 4 \tau_{f}\right)}{\left(\pi \tau_{f}\right)^{1 / 2}}=\frac{Y_{\infty}}{Y_{\infty}+F_{i}} .
$$

The corresponding two-dimensional result for a cylinder of radius $a$ is [2]

$$
\tau_{f}=\left[4 \ln \left(\frac{Y_{\infty}+F_{i}}{F_{i}}\right)\right]^{-1} .
$$

In fact, the generalization of (4.12a) to include the effect of a strain rate, constant in space and time, along the axis of the cylinder,

$$
w_{z}(r, z, t)=\varepsilon_{0},
$$

is shown in Appendix $\mathrm{C}$ to be

$$
\frac{\exp \left[4 \bar{\varepsilon} \tau_{f}\right]-1}{\bar{\varepsilon}}=\frac{1}{\ln \left[\left(Y_{\infty}+F_{i}\right) / F_{i}\right]}
$$

where

$$
\bar{\varepsilon}=\frac{\varepsilon_{0} a^{2}}{4 D_{\infty}} .
$$

In these problems, a large strain rate would imply a rapid evolution to a flat geometry, so it is of interest here to treat the consumption of a strip of fuel with a finite strain rate, i.e., with $\varepsilon \neq 0$.

where the argument of the exponential is constant in time from (4.9), so the temporal dependence lies entirely in $r(t)$. For $\varepsilon$ constant, from (4.2) and (4.4),

$$
r^{2}=r_{0}^{2} \exp (-2 \varepsilon t)+(2 / \varepsilon)[1-\exp (-2 \varepsilon t)]
$$

for $r_{0} \rightarrow 0$, the reactant consumption rate is, in dimensional terms, proportional to $\rho_{\infty}\left(D_{\infty} / t\right)^{1 / 2}$ for $t \rightarrow 0$, and hence independent of $\varepsilon$, and proportional to $\rho_{\infty}\left(D_{\infty} \varepsilon\right)^{1 / 2}$ for $t \rightarrow \infty$. 


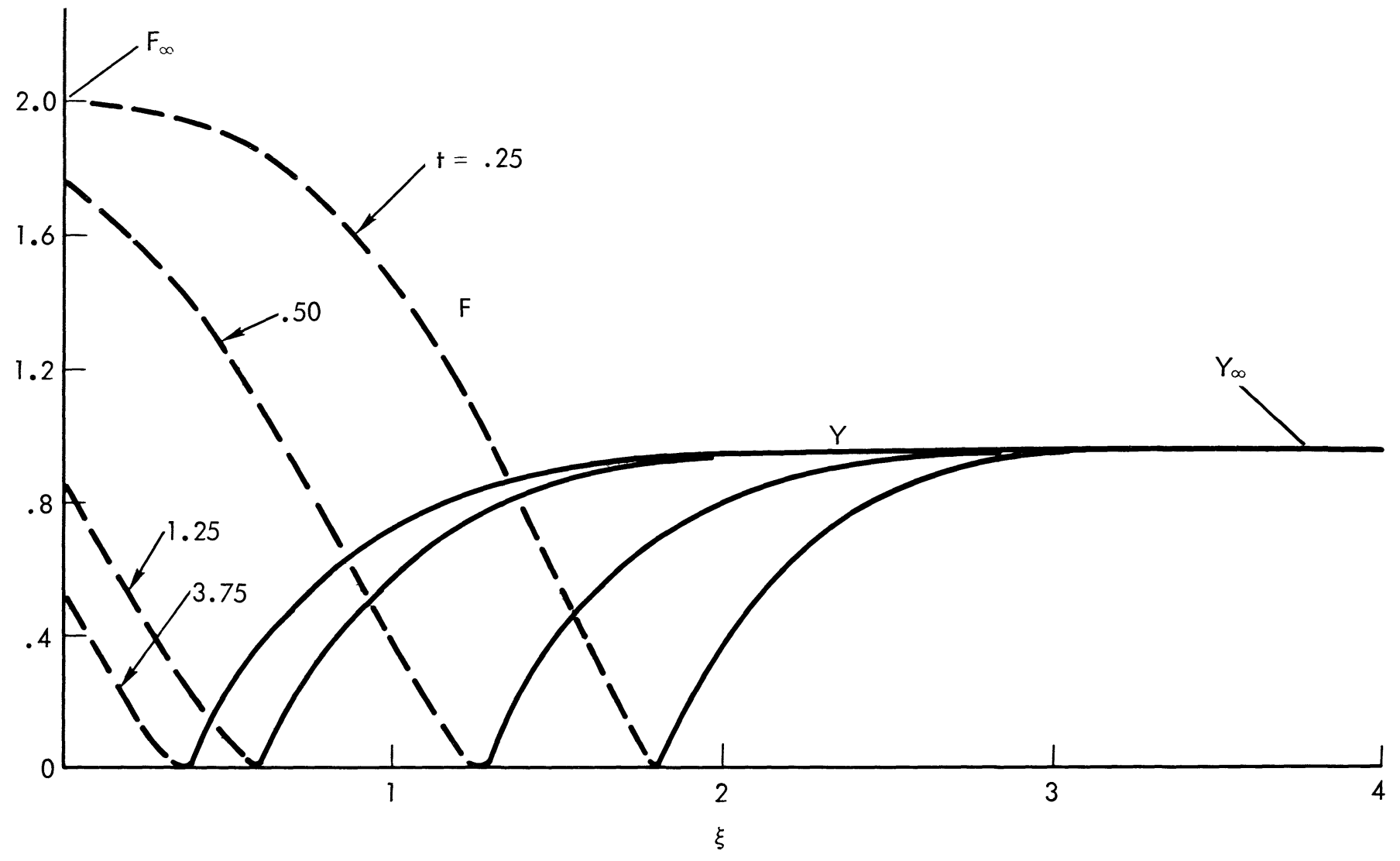

FIG. 3. The self-similar profiles of the stoichiometrically adjusted mass fractions for fuel (dashed lines) and oxidant (solid lines) for unsteady equilibrium irreversible burning in a counterflow. For $Y_{\infty}=0.95, F_{\infty}=2.0, h_{\infty}=0.5, h_{-\infty}=0.1, Y$ and $F$ are given for times $t=0.25,0.50,1.25$ and $3.75($ the last closely approximates the solution for $t \rightarrow \infty)$. The evolution for single flame is from an initial condition $(t=0)$ for which $h=0.5, F=0, Y=0.95$ for $\xi>2.5 ; h=0.1, F=2.0$, and $Y=0$ for $\xi<2.5$. Since $\varepsilon(\tau)=2.0, m_{0}=2.5$, and $r_{0}=0.01$, the asymptotic solution as $t \rightarrow \infty$ is that given in Fig. 2 


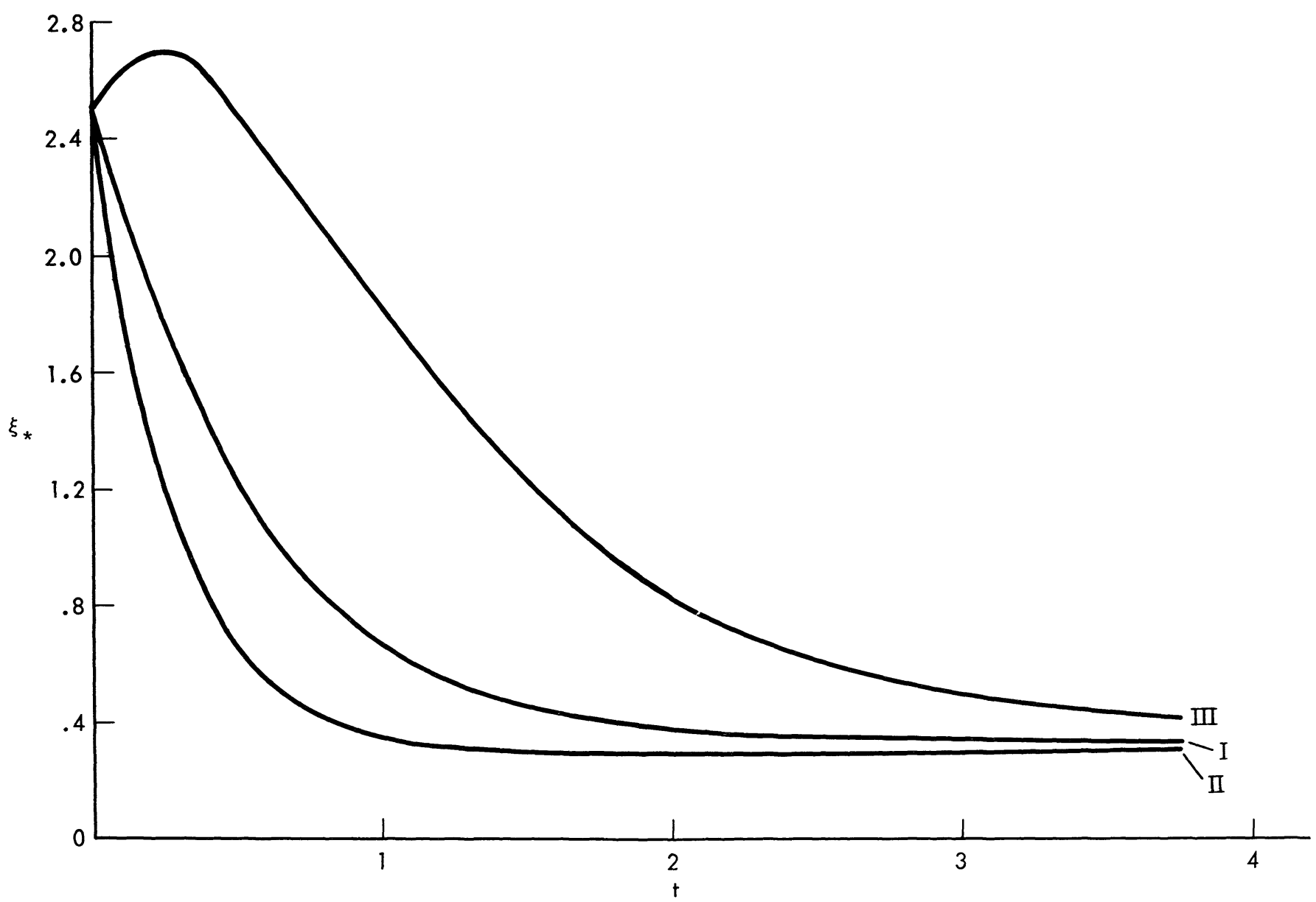

FiG. 4. The position $\xi_{*}$ as a function of time $t$ of a single thin flame evolving from the initial conditions, and subject to the boundary conditions given in the caption to Fig. 3. For all cases at all times, the adiabatic flame enthalpy $h_{*}=1.015$. The cases are differentiated by values of $\varepsilon(t)$ : I. $\varepsilon=2$; II. $\varepsilon=2+2 /(1+t) ;$ III. $\varepsilon=2 /(1+t)$ 
The burning off of a strip of fuel by surrounding oxidant models is what may transpire in region 2 of Fig. 1. The foregoing study of the transient single flame furnishes virtually all of the analysis required for current purposes. As a model of the phenomenon, we postulate that [cf. (2.7b)]

$$
\begin{aligned}
& Y(\xi, 0)= \begin{cases}Y_{\infty}, & |\xi|>m_{0}, \\
0, & |\xi|<m_{0},\end{cases} \\
& F(\xi, 0)= \begin{cases}0, & |\xi|>m_{0}, \\
F_{i}, & |\xi|<m_{0},\end{cases} \\
& h(\xi, 0)= \begin{cases}h_{\infty}, & |\xi|>m_{0}, \\
h_{i}, & |\xi|<m_{0} .\end{cases}
\end{aligned}
$$

The combinations which have the form of (4.5) and which are consistent with the initial conditions (4.13) are

$$
\begin{aligned}
& Y-F=Y_{\infty}+\frac{F_{i}+Y_{\infty}}{2}\left\{\operatorname{erf}\left[\frac{\xi-m(t)}{r(t)}\right]-\operatorname{erf}\left[\frac{\xi+m(t)}{r(t)}\right]\right\}, \\
& Y+h=Y_{\infty}+h_{\infty}+\frac{Y_{\infty}+h_{\infty}-h_{i}}{2}\left\{\operatorname{erf}\left[\frac{\xi-m(t)}{r(t)}\right]-\operatorname{erf}\left[\frac{\xi+m(t)}{r(t)}\right]\right\},
\end{aligned}
$$

where $r(t)$ and $m(t)$ are given by (4.2) and (4.3), respectively, with $r_{0} \rightarrow 0$. Flame fronts will lie at $\pm \xi_{*}(t)$, such that $F=0$ for $\xi \geqq\left|\xi_{*}(t)\right|$ and $Y=0$ for $\xi \leqq\left|\xi_{*}(t)\right|$, where $\xi_{*}(t)$ may be found by setting the right-hand side of (4.14a) to zero. Following the procedure of (4.8), the position of the flames for $t>0$ may be determined from

$$
\begin{aligned}
\frac{d \xi_{*}}{d t}= & -\frac{\left\{m \varepsilon-\left(\xi_{*}-m\right)(\dot{d} / \dot{d} t)(\ln r)\right\}}{1-\exp \left(-4 m \xi_{*} / r^{2}\right)} \\
& -\frac{\left\{\exp \left[-4 m \xi_{*} / r^{2}\right]\right\}\left\{m \varepsilon+\left(\xi_{*}+m\right)(d / d t)(\ln r)\right\}}{1-\exp \left(-4 m \xi_{*} / r^{2}\right)},
\end{aligned}
$$

where $\xi_{*}(t=0)= \pm m_{0}$. Note that $\xi_{*}(t)$ becomes zero, i.e., all the fuel is consumed, at a finite time $t_{f}$, where, from (4.14a),

$$
\operatorname{erf}\left[\frac{m\left(t_{f}\right)}{r\left(t_{f}\right)}\right]=\frac{Y_{\infty}}{F_{i}+Y_{\infty}} .
$$

The approach to extinction, from (4.15), is

$$
\xi_{*} \simeq\left[2\left(t_{f}-t\right)\right]^{1 / 2} \text {. }
$$

For $t>t_{f},(4.14)$ continues to furnish the solution, with $F=0$. Results depicting consumption of the fuel and subsequent behavior are depicted in Figs. 5 and 6.

For $1 \gg D_{1}^{-1}>0$, each flame has a thin but finite structure whose thickness is of order $D_{1}^{-1 /\left(1+v_{0}+v_{F}\right)}$, similar to that described above for the single timedependent flame. When $\left|\xi_{*}\right|$ is of order $D_{1}^{-1 /\left(1+v_{0}+v_{F}\right)}$, the two flames interact. At the onset of interaction, the mass fraction of fuel remaining is of order $D_{1}^{-2 /\left(1+v_{0}+v_{F}\right)}$. 


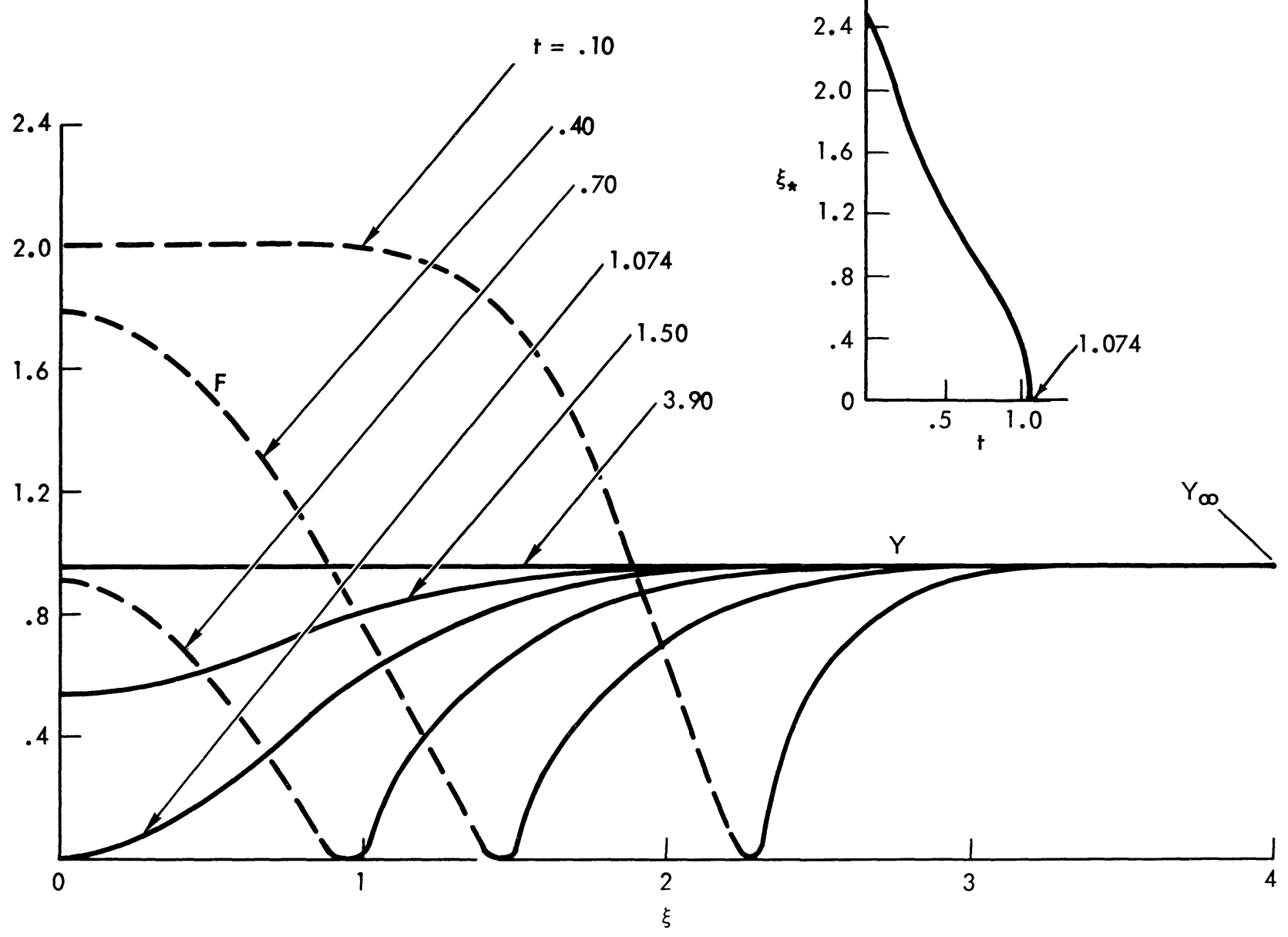

FIG. 5. The consumption of a fuel strip in time by surrounding oxidant via a one-step equilibrium irreversible reaction. Because the solution for this case is symmetric about $\xi=0$ at all times, only the upper half-plane is shown. The spatially uniform initial conditions $(t=0)$ for the plotted case were $Y=0.95, F=0, h=0.5$ for $|\xi|>2.5 ; Y=0, F=2, h=0.1$ for $|\xi|<2.5$. The boundary conditions were $Y \rightarrow Y_{\infty}=0.95$, $F \rightarrow 0, h \rightarrow h_{\infty}=0.5$ for $|\xi| \rightarrow \infty$. Here $\varepsilon(t)=2.0, m_{0}=2.5$, and $r_{0}=0.01$. The progress of the thin flame position $\xi_{*}$ in time $t$ is further elucidated by the insert. After all the fuel is consumed at $t \doteq 1.074$, the oxidant proceeds to fill the entire space uniformly. 


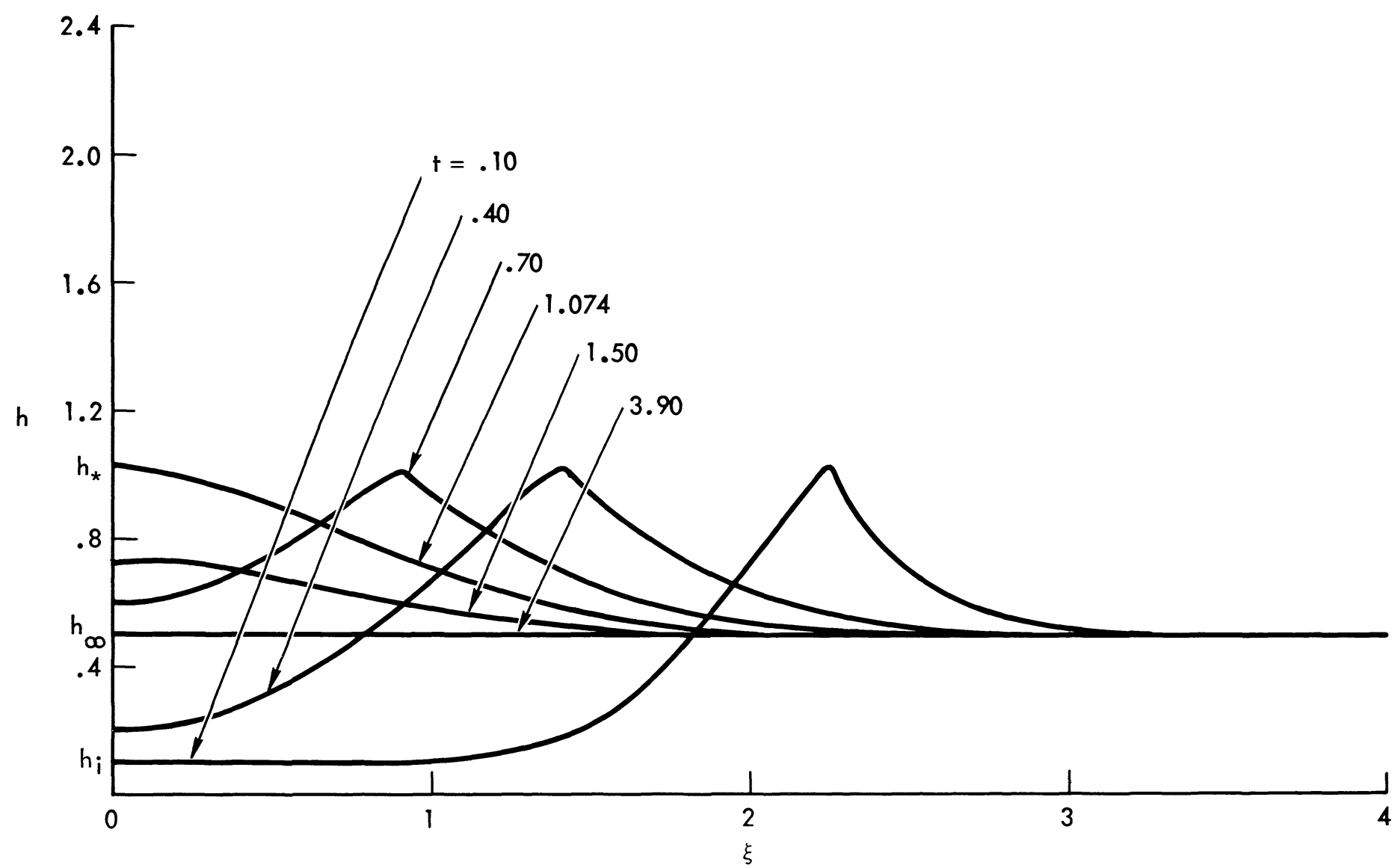

FIG. 6. The enthalpy profiles associated with the boundary value problem plotted in Fig. 5. The ambient enthalpy is $h_{\infty}$; the initial fuel enthalpy is $h_{i}$. The adiabatic flame enthalpy $h_{*}=1.015$ and is achieved at the thin flame at all times $t$ for which fuel is present; after all fuel is consumed at $t \doteq 1.074$, the enthalpy gradually decreases to be uniform throughout the space at the ambient value. 
This supply of fuel goes to zero exponentially in time; the time constant is of order $D_{1}^{-2 /\left(1+v_{0}+v_{F}\right)}$.

For purposes of comparison, by (4.2)-(4.4), (4.16) may be rewritten

$$
\operatorname{erf}\left[\bar{\varepsilon}^{-1} \int_{0}^{4 \bar{\varepsilon} \tau_{f}} \exp \left[2 v\left(t_{1}\right)\right] d t_{1}\right]^{-1 / 2}=\frac{Y_{\infty}}{Y_{\infty}+F_{i}}
$$

where $m_{0}$, the half-width of the strip, has replaced the radius $a$ in the definition of $\bar{\varepsilon}$, given below ( $4.12 \mathrm{~b}$ ), and $\varepsilon_{0}$ characterizes the Cartesian (rather than cylindrical) strain rate. Equation (4.18a) holds for temporally variant strain rate ; for $\varepsilon$ constant, (4.18a) becomes

$$
\operatorname{erf}\left[\frac{\exp \left(8 \bar{\varepsilon} \tau_{f}\right)-1}{2 \bar{\varepsilon}}\right]^{-1 / 2}=\frac{Y_{\infty}}{Y_{\infty}+F_{i}},
$$

and for $\varepsilon=0,(4.18 \mathrm{a})$ becomes [cf. (4.11)]

$$
\operatorname{erf}\left[\frac{1}{2 \tau_{f}^{1 / 2}}\right]=\frac{Y_{\infty}}{Y_{\infty}+F_{i}} .
$$

A comparison of the times for consumption, as a function of $\left(Y_{\infty} / F_{i}\right)$, in the absence of a strain, for a sphere, cylinder, and strip (as obtained from (4.11), (4.12a), and (4.18c), respectively) is given in Fig. 7. Incidentally, the spatial region occupied by fuel need not monotonically decrease in time; for example, it is known that for a sphere of fuel, the position of the flame initially increases in time if $Y_{\infty}<F_{i}$. If $Y_{\infty}=F_{i}$, the fuel consumption time owing to finite strain rate is halved for a cylinder for a strain rate such that $\bar{\varepsilon} \doteq 1.74$ according to $(4.12 \mathrm{~b})$, and is halved for strip for $\bar{\varepsilon} \doteq 0.286$ according to (4.18b).

5. Concluding remarks. In a nonstraining laminar mixing layer, the first Damköhler number (the ratio of a flow time to a reaction time characterized by the specific rate constant) increases linearly with distance downstream, since the flow time is characterized by the ratio of the distance from the start of the layer to a typical local speed of the streams. In contrast to this, the flow time which helps to define the first Damköhler number associated with a straining laminar flow is the reciprocal of the strain rate. The straining of the flame is physically significant because it increases the rate of reactant consumption, not only by increasing the interfacial exposure of fuel to oxidant, but also by convecting additional reactant to the flame.

The magnitude of the correction to the Burke-Schumann thin-flame solution in the outer regions away from the flame zone, under near-equilibrium conditions depends on stoichiometry. For example, as the order of the reaction with respect to fuel passes from less than unity, to precisely unity, to greater than unity, the magnitudes of the corrections on the oxidant-rich side of the burning zone increase from zero, to exponentially small, to algebraically small, in their functional dependence on the inverse of the first Damköhler number $D_{1}$ as $D_{1} \rightarrow \infty$.

Ordinarily, the small but finite flame structure in unpremixed reactants for fast but finite reaction rate relative to flow rate may be described to the leading order of approximation by a quasi-steady theory in which time enters parametrically only. The situation becomes more complex when such independently derived 


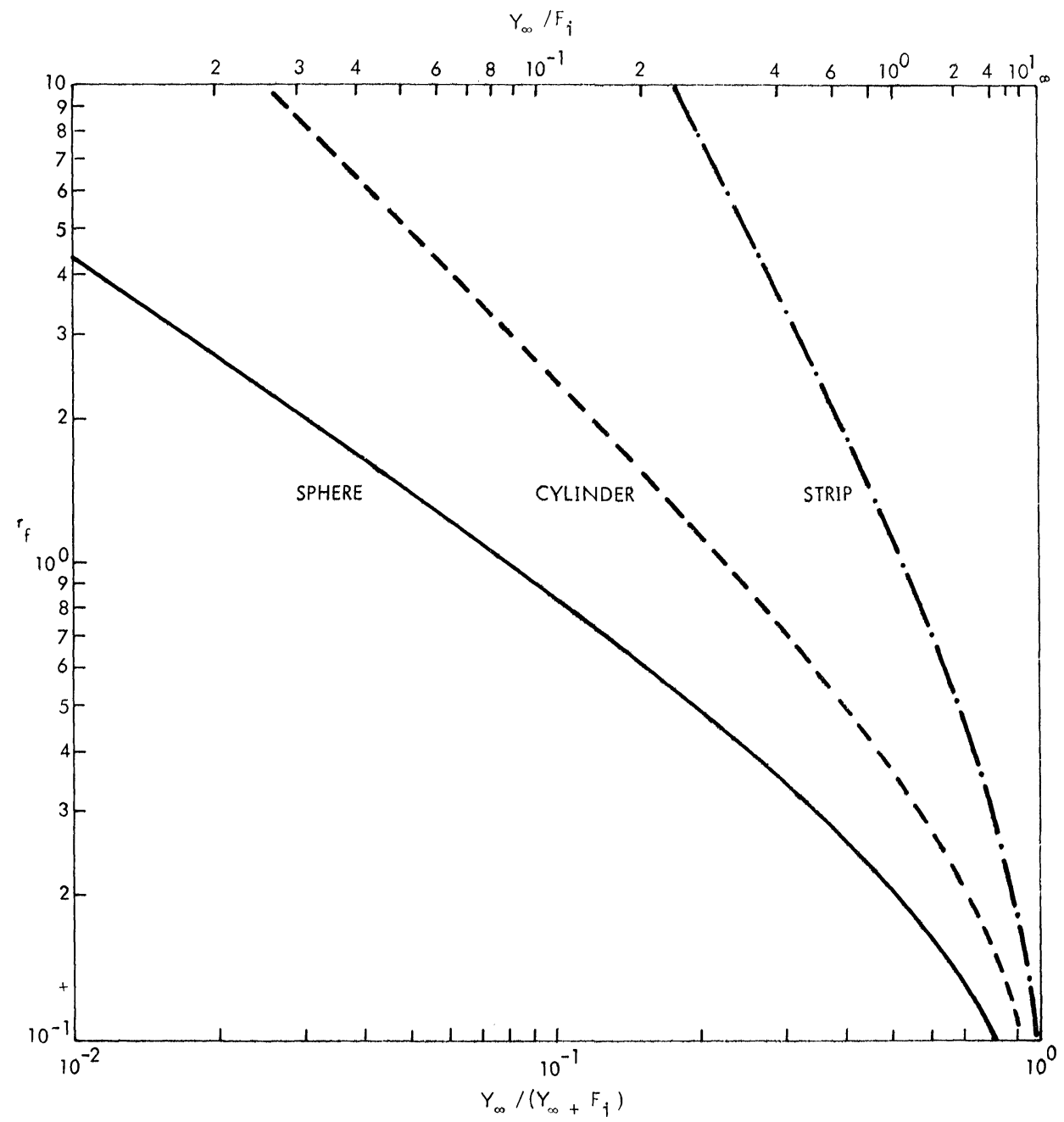

FIG. 7. The time for consumption of a volume of fuel in an oxidant atmosphere is given as a function of stoichiometry $Y_{\infty} / F_{i}$ only, for a one-step equilibrium irreversible reaction in the absence of strain. The burning time $\tau_{f}\left(\equiv t_{f} D_{\infty} / a^{2}\right)$ is given for a sphere of radius a by (4.11), for a cylinder of radius a by (4.12a), and for a strip of half-width a by $(4.18 \mathrm{c})$.

flame structures are found to interact because extensive straining has narrowed the intervening region containing a combustible. In particular, a narrow fuel strip separated from surrounding oxidant by an encompassing diffusion flame is entirely consumed in a finite time, according to a time-dependent solution based on the thin-flame limit. In the more realistic flame, all of the fuel except an amount of order $D_{1}^{-1}$ (for $v_{0}=v_{F}=1$ ) is consumed in that same time; the remaining fuel supply is of order $D_{1}^{-1} \exp \left[-t / t_{0}\right]$. Thus, for all practical purposes, the thin flame theory suffices to describe the local extinction process. 
Appendix A. The steady near-equilibrium irreversible limit. If $1 \gg D_{1}^{-1}>0$, as opposed to the case $D_{1} \rightarrow \infty$ treated in $\S 3$, then the boundary-value problem $\left[\zeta=(\varepsilon / 2)^{1 / 2} \xi\right]$ is given by

$$
L_{1}(h)=\left\{\frac{d^{2}}{d \zeta^{2}}+2 \zeta \frac{d}{d \zeta}\right\} h=-D_{1} Y^{\nu_{0}} F^{v_{F}} \exp \left(-\frac{\theta}{h+\alpha}\right),
$$

$$
L_{1}(Y+h)=L_{1}(F+h)=0,
$$

where the boundary conditions given in $\S 3$ still hold. Equations (3.1)-(3.3) constitute exact integrals of (A.2) for any $D_{1}$; any local asymptotic series expansions (for $1 \gg D_{1}^{-1}>0$, say) for $Y, F$, and $h$ must be compatible with (3.1)-(3.3).

Asymptotic expansions are now postulated, to be later confirmed by matching, for a three-region subdivision of the flow field for $v_{0}>1, v_{F}>1$. As shown in Fig. 8, there is an upstream oxidant zone, a downstream fuel zone, and an intermediate reaction, thin relative to the thickness of the other two zones.

Upstream oxidant zone $\left(v_{F}>1\right)$ :

$$
\begin{aligned}
& F(\zeta ; \delta)=\quad \delta^{1 /\left(v_{F}-1\right)} F_{1}(\zeta)+\cdots ; \\
& Y(\zeta ; \delta)=Y_{0}(\zeta)+\delta^{1 /\left(v_{F}-1\right)} Y_{1}(\zeta)+\cdots ; \\
& h(\zeta ; \delta)=H_{0}(\zeta)+\delta^{1 /\left(v_{F}-1\right)} H_{1}(\zeta)+\cdots .
\end{aligned}
$$

Downstream fuel zone $\left(v_{0}>1\right)$ :

$$
F(\zeta ; \delta)=f_{0}(\zeta)+\delta^{1 /\left(v_{0} 1\right)} f_{1}(\zeta)+\cdots ;
$$

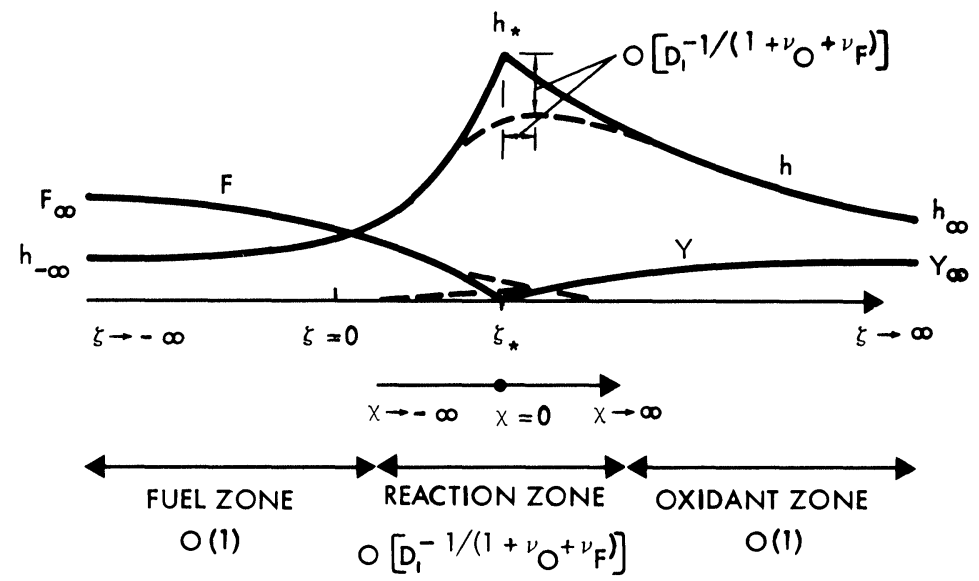

FIG. 8. A schematic view of the perturbation from thin-flame conditions to small but finite flame structure in initially unmixed reactants for one-step chemistry. If $\zeta$ is a spatial coordinate locally perpendicular to the flame (located at $\zeta_{*}$ ), and if the solid lines denote the profiles of $h, F$, and $Y$ for equilibrium irreversible burning, then the dashed lines give the near-equilibrium perturbation for finite structure [large but finite Damköhler number $D_{1}$ ]. The thickness and the displacement from $\zeta_{*}$ and decrement from $h_{*}$ (the adiabatic flame enthalpy) of the maximum enthalpy, are all $O\left[D_{1}^{-1 /\left(1+v_{0}+v_{F}\right)}\right]$, where $v_{0}, v_{F}$ are the stoichiometric coefficients for oxidant and fuel, respectively. A scaled coordinate $\chi$ with origin at $\zeta_{*}$ is introduced for reaction-zone analysis. 
(A.4b)

$$
\begin{aligned}
& Y(\zeta ; \delta)=\quad \delta^{1 /\left(v_{0}-1\right)} y_{1}(\zeta)+\cdots ; \\
& h(\zeta ; \delta)=h_{0}(\zeta)+\delta^{1 /\left(v_{0}-1\right)} h_{1}(\zeta)+\cdots
\end{aligned}
$$

Intermediate reaction zone $\left[\chi=\left(\zeta-\zeta_{*}\right) / \delta^{b}, b=1 /\left(1+v_{0}+v_{F}\right)\right]$ :

$$
\begin{aligned}
& F(\zeta ; \delta)=\delta^{b} \mathscr{F}_{0}(\chi)+\delta^{2 b} \mathscr{F}_{1}(\chi)+\cdots \\
& Y(\zeta ; \delta)=\delta^{b} \mathscr{Y}_{0}(\chi)+\delta^{2 b \mathscr{Y}_{1}}(\chi)+\cdots ; \\
& h(\zeta ; \delta)=h_{*}-\delta^{b} \mathscr{H}_{0}(\chi)+\delta^{2 b} \mathscr{H}_{1}(\chi)+\cdots
\end{aligned}
$$

The expansion parameter

$$
\delta=\left\{D_{1} \exp \left[-\theta /\left(h_{*}+\alpha\right)\right]\right\}^{-1}, \quad \delta \ll 1 .
$$

The quantity $\zeta_{*}$ occurring in $\chi$ is given by (3.4); $h_{*}$ is given by (3.5). If $\theta$ were large, the lowest order terms would still be valid provided $D_{1}$ were so large that $\delta \ll 1$. However, the higher order terms, given here for $\theta=O(1)$ and $\delta \ll 1$, would need revision.

The anticipated form for the asymptotic expansions is based on previous work. The lowest order terms in all zones were given by Pearson [13], Friedlander and Keller [8], Liñan [12], and Fendell [6]. Fendell [7] also gave the higher order terms in the reaction zone and noted the absence of higher order algebraically large corrections in the oxidant zone if $v_{F}=1$, and in the oxidant zone if $v_{0}=1$ (the upstream and/or downstream corrections in these cases are of exponentially small order, as implied by the asymptotic behavior of the lowest order reactionzone solution, given below). The explicit ordering of the higher order upstream (downstream) terms for $v_{F}\left(v_{0}\right)$ greater than unity was given by Clarke [3], [4] and by Kassoy, Liu, and Williams [10], [11]. The nature of the higher order terms in the upstream (downstream) expansions for $v_{F}<1\left(v_{0}<1\right)$ is stated to be unresolved by Williams [17]; the behavior will be resolved here. Finally, numerical results for the nonlinear boundary value problem describing the lowest order reaction-zone solution ${ }^{5}$ are presented which are more extensive than any previously given.

Substitution of (A.3) and (A.4) in (A.1) and (A.2) should yield the so-called Burke-Schumann solution, (3.1)-(3.3), to lowest order of approximation:

$$
\begin{aligned}
& Y_{0}+H_{0}=c_{1} \operatorname{erf} \zeta+c_{2}, \quad H_{0}=c_{3} \operatorname{erf} \zeta+c_{4}, \\
& h_{0}=c_{1} \operatorname{erf} \zeta+c_{2}, \quad f_{0}+h_{0}=c_{3} \operatorname{erf} \zeta+c_{4}
\end{aligned}
$$

A.1. The reaction zone $\left(v_{0} \geqq 1, v_{F} \geqq 1\right)$. Substitution of (A.5) in (A.1) and (A.2) (boundary conditions are replaced by requirements of matching to the upstream and downstream expansions) gives to lowest order of approximation:

$$
\begin{aligned}
& \frac{d^{2}}{d \chi^{2}}\left(\mathscr{F}_{0}-\mathscr{H}_{0}\right)=0 \Rightarrow \mathscr{F}_{0}-\mathscr{H}_{0}=a_{0} \chi+b_{0} \\
& \frac{d^{2}}{d \chi^{2}}\left(\mathscr{Y}_{0}-\mathscr{H}_{0}\right)=0 \Rightarrow \mathscr{Y}_{0}-\mathscr{H}_{0}=c_{0} \chi+d_{0}
\end{aligned}
$$

${ }^{5}$ The following oversights in [7] are noted: Figure 4 has an error corrected below; also $b$ and $d$ should be interchanged in (27) because $\bar{y}_{F_{0}} \sim(U-x)$ and $\bar{y}_{0_{0}} \sim(U+x)$. 


$$
\frac{d^{2} \mathscr{H}_{0}}{d \chi^{2}}=\left(\mathscr{F}_{0}\right)^{v_{F}}\left(\mathscr{H}_{0}\right)^{v_{0}}
$$

Matching the inner and outer expansions for $(Y+h)$ and $(F+h)$ gives (see (3.1)(3.4))

$$
c_{0}=\frac{2 c_{1}}{\pi^{1 / 2}} \exp \left(-\zeta_{*}^{2}\right), \quad a_{0}=\frac{2 c_{3}}{\pi^{1 / 2}} \exp \left(-\zeta_{*}^{2}\right)
$$

Further, $b_{0}=d_{0}=0$ by comparing the local Shvab-Zeldovich-function expansions (i.e., the asymptotic expansion implied by (A.5), (A.9), and (A.10) for $(Y+h)$ and $(F+h)$ in the limit $\delta \ll 1)$ with the known exact expressions for these functions given by (3.1)-(3.5).

$$
\text { If } \text { (A.13) } U(\psi) \equiv Q\left(\mathscr{H}_{0}+\frac{a_{0}+c_{0}}{2} \chi\right), \quad \psi \equiv Q\left(\frac{c_{0}-a_{0}}{2}\right) \chi, \quad Q \equiv\left(\frac{2}{c_{0}-a_{0}}\right)
$$

then (A.9)-(A.11) becomes universal in form (except for $\left.v_{0}, v_{F}\right)$ :

$$
\frac{d^{2} U}{d \psi^{2}}=(U-\psi)^{v_{F}}(U+\psi)^{v_{0}}
$$

where matching requires that

$$
\psi \rightarrow-\infty, \quad U \rightarrow-\psi ; \quad \psi \rightarrow \infty, \quad U \rightarrow \psi
$$

the boundary conditions reflect the vanishing of fuel (oxidant) upstream (downstream), to lowest order of approximation. The boundary value problem (A.14) has the following properties: (a) $U=|\psi|$ is the (nonanalytic) Burke-Schumann solution, which is independent of $v_{0}$ and $v_{F}$, but which is unacceptable here; (b) $U\left(\psi ; v_{0}, v_{F}\right)$ is a solution, then $U\left(-\psi ; v_{F}, v_{0}\right)$ is also a solution; (c) if $\beta=v_{0}=v_{F}$, then $U\left(\psi ; v_{0}, v_{F}\right)=U\left(\psi^{2} ; \beta, \beta\right)$, i.e., the solution is even in $\psi$, so one of the boundary conditions (A.14b) may be conveniently replaced by $d U(\psi=0, \beta, \beta) / d \psi=0$; and $(\mathrm{d})$ at all finite $\psi$, since $(U-\psi)$ and $(U+\psi)$ are positive definite on physical grounds, $d^{2} U / d \psi^{2}>0$, and thus $U$ has one and only one extremum, a minimum (which occurs at $\psi=0$ for $v_{0}=v_{F}=\beta$ ). Numerical results for various $v_{0}, v_{F}$, obtained by quasi-linearization techniques, are given in Figs. 9-13.

From (3.2), (3.3), (A.5c), (A.12), and (A.13), the maximum enthalpy, decremented from the adiabatic flame temperature $h_{*}$, occurs at $\psi_{m}$, where

$$
\frac{d U\left(\psi_{m}\right)}{d \psi}=\frac{c_{0}+a_{0}}{c_{0}-a_{0}}=\frac{c_{1}+c_{3}}{c_{1}-c_{3}}=\frac{Y_{\infty}-F_{\infty}+2\left(h_{\infty}-h_{-\infty}\right)}{Y_{\infty}+F_{\infty}} .
$$

This result is based on the first two terms only of the expansion given in (A.5c). In general, the maximum is displaced from its thin-flame position $\psi=0$. The maximum enthalpy itself is

$$
h_{m}=h_{*}-\frac{\varepsilon^{b}}{Q}\left[U\left(\psi_{m}\right)-\psi_{m} \frac{d U\left(\psi_{m}\right)}{d \psi}\right]+O\left(\varepsilon^{2 b}\right),
$$

where the expression in square brackets must be positive definite for all finite $\psi_{m}$ for a solution to (A.14) to exist.

For $v_{0}$ arbitrary, $v_{F}=1$, one seeks the asymptotic behavior of $U$ as $\psi \rightarrow \infty$ by substituting $U=\psi+f\left(\psi ; v_{0}\right)$ and linearizing in $f$. One finds the exponentially 
small asymptotic behavior for $f$ anticipated earlier [13]:

$$
\frac{d^{2} f}{d \psi^{2}}-(2 \psi)^{v_{0}} f=0 \Rightarrow f \sim \psi^{-v_{0} / 4} \exp \left\{-\frac{(2 \psi)^{\left(v_{0}+2\right) / 2}}{v_{0}+2}\right\}
$$

letting $U=-\psi+g$ for $v_{0}=1, v_{F}$ arbitrary, yields, as $\psi \rightarrow-\infty$,

$$
g \sim(-\psi)^{-v_{F} / 4} \exp \left\{-\frac{[2(-\psi)]^{\left(v_{F}+2\right) / 2}}{v_{F}+2}\right\} .
$$

More generally, for $\psi \rightarrow \infty$, for arbitrary $v_{0}+v_{F}$, the asymptotic behavior of (A.14) may be found by letting $U=\psi+f$; without linearizing,

$$
\frac{d^{2} f}{d \psi^{2}}=f^{v_{F}}(2 \psi+f)^{v_{0}} .
$$

For $v_{F}>1$, an algebraic decay [3], [4], [10], [11] anticipated earlier arises :

$$
f=A \psi^{k}+B \psi^{s}+\cdots, \quad 1>k>s .
$$

Substituting (A.20) in (A.19) and equating coefficients of equal powers of $\psi$, one finds

$$
\begin{aligned}
& A=\left[\frac{k(k-1)}{2^{v_{0}}}\right]^{1 /\left(v_{F}-1\right)}, \quad k=-\frac{v_{0}+2}{v_{F}-1}, \\
& B=\frac{A^{2} k v_{0}}{2\left[\left(4-v_{F}\right) k-2\right]}, \quad s=2 k-1 .
\end{aligned}
$$

Again, as $\psi \rightarrow-\infty$, for $v_{0}>1, U=(-\psi)+g$, where

$$
g=A_{1}(-\psi)^{k_{1}}+B_{1}(-\psi)^{s_{1}}+\cdots, \quad 1>k_{1}>s_{1},
$$

and

$$
\begin{aligned}
& A_{1}=\left[\frac{k_{1}\left(k_{1}-1\right)}{2^{v_{F}}}\right]^{1 /\left(v_{0}-1\right)}, \quad k_{1}=-\frac{v_{F}+2}{v_{0}-1}, \\
& B_{1}=\frac{A_{1}^{2} k_{1} v_{F}}{2\left[\left(4-v_{0}\right) k_{1}-2\right]}, \quad s_{1}=2 k_{1}-1 .
\end{aligned}
$$

For convenience the asymptotic forms of the dependent variables as $\chi \rightarrow \infty$ for $v_{F}>1$, and as $\chi \rightarrow-\infty$ for $v_{0}>1$, are presented. As $\chi \rightarrow \infty$,

$$
\begin{aligned}
h \sim h_{*}-\delta^{b}\{ & -a_{0} \chi+e_{0} \chi^{-\left(v_{0}+2\right) /\left(v_{F}-1\right)} \\
& \left.+O\left[\chi^{-\left(3+2 v_{0}+v_{F}\right) /\left(v_{F}-1\right)}\right]\right\}+O\left(\delta^{2 b}\right)
\end{aligned}
$$

$$
F \sim \delta^{b}\left\{e_{0} \chi^{-\left(v_{0}+2\right) /\left(v_{F}-1\right)}\right.
$$

$$
\left.+O\left[\chi^{-\left(3+2 v_{0}+v_{F}\right) /\left(v_{F}-1\right)}\right]\right\}+O\left(\delta^{2 b}\right)
$$

$$
\begin{aligned}
& Y \sim \delta^{b}\left\{\left(c_{0}-a_{0}\right) \chi+e_{0} \chi^{-\left(v_{0}+2\right) /\left(v_{F}-1\right)}\right. \\
& \left.+O\left[\chi^{-\left(3+2 v_{0}+v_{F}\right) /\left(v_{F}-1\right)}\right]\right\}+O\left(\delta^{2 b}\right)
\end{aligned}
$$




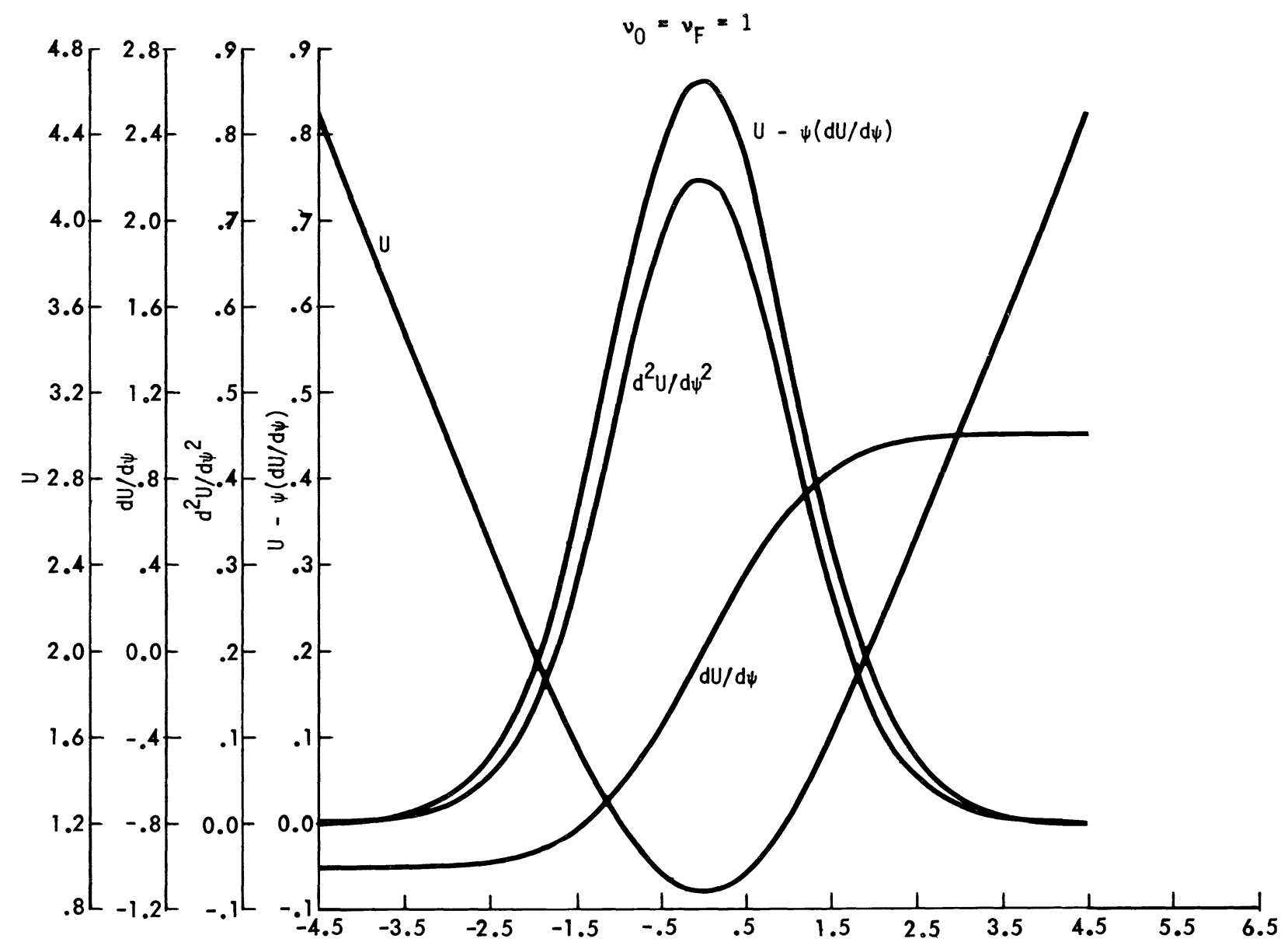

FIG. 9. Results of numerical integration of the nonlinear boundary value problem (A.14) describing the finite reaction zone to lowest order: $d^{2} U / d \psi^{2}=(U-\psi)^{v_{F}}(U+\psi)^{\nu_{0}}, U(\psi \rightarrow \infty) \rightarrow \psi, U(\psi \rightarrow-\infty) \rightarrow-\psi$. For $v_{0}=v_{F}=1, U(\psi=0)=0.86570 \cdots, d U(\psi=0) / d \psi=0$. 


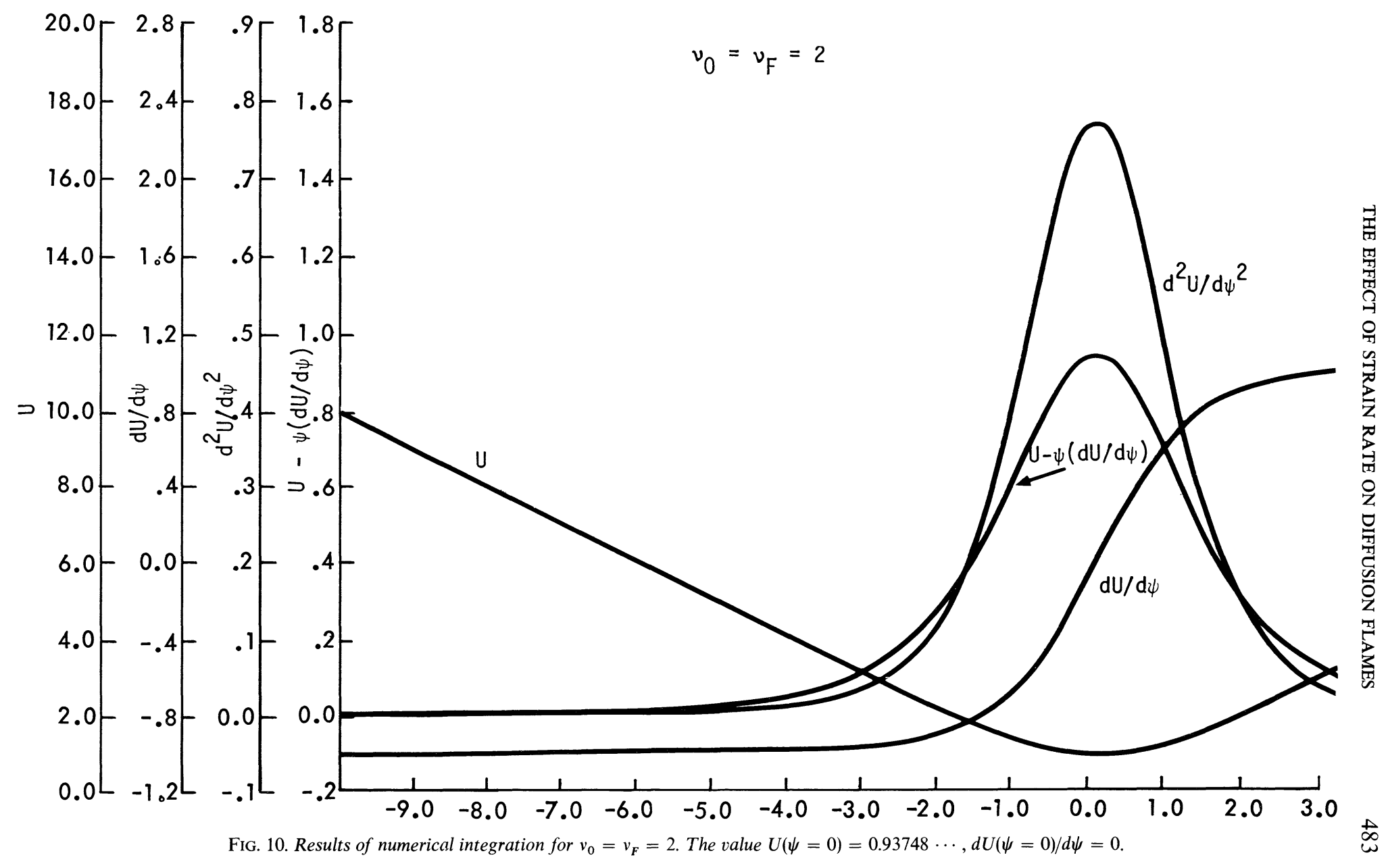




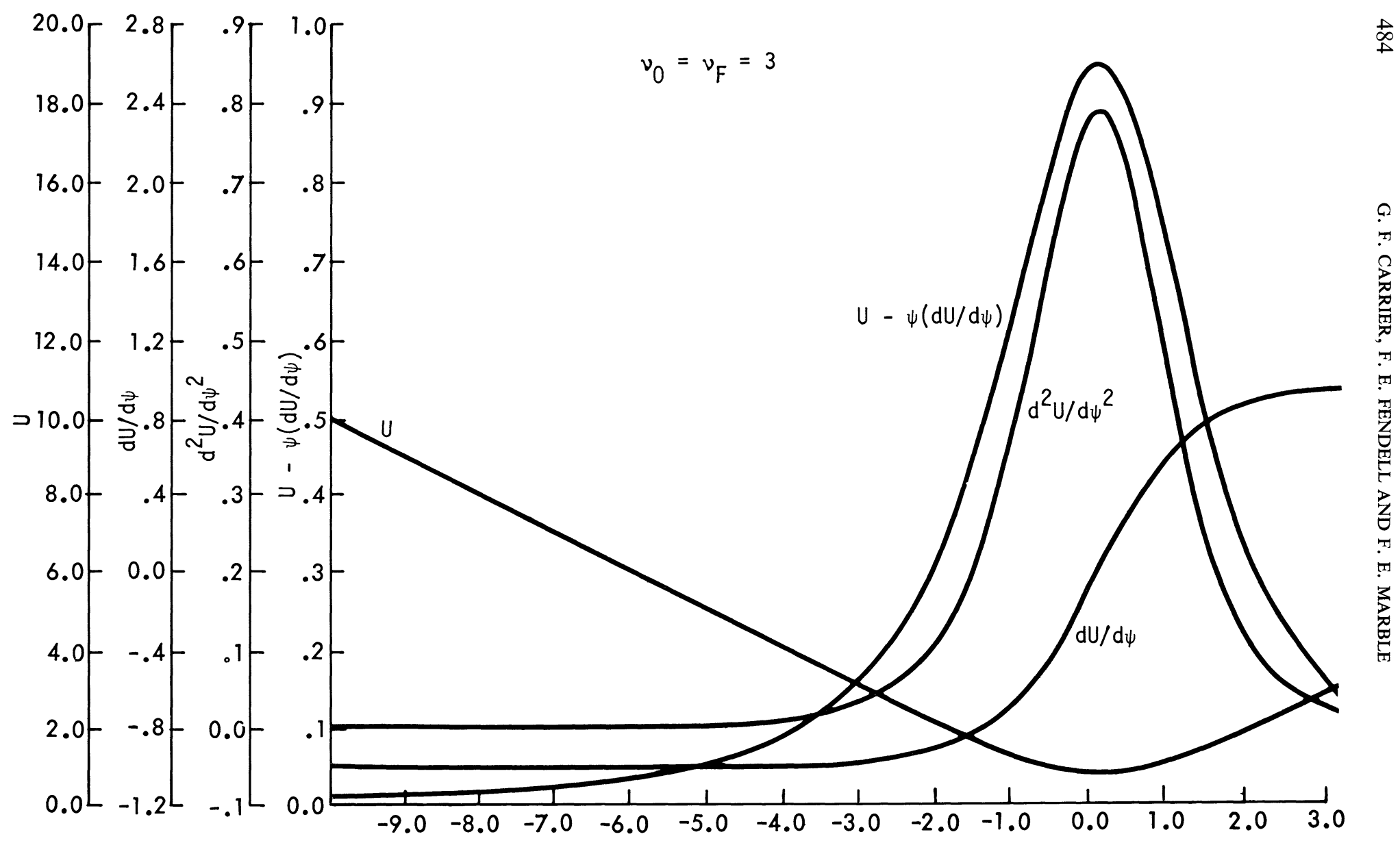

Fig. 11. Results of numerical integration for $v_{0}=v_{F}=3$. The value $U(\psi=0)=0.96355 \cdots, d U(\psi=0) / d \psi=0$. 


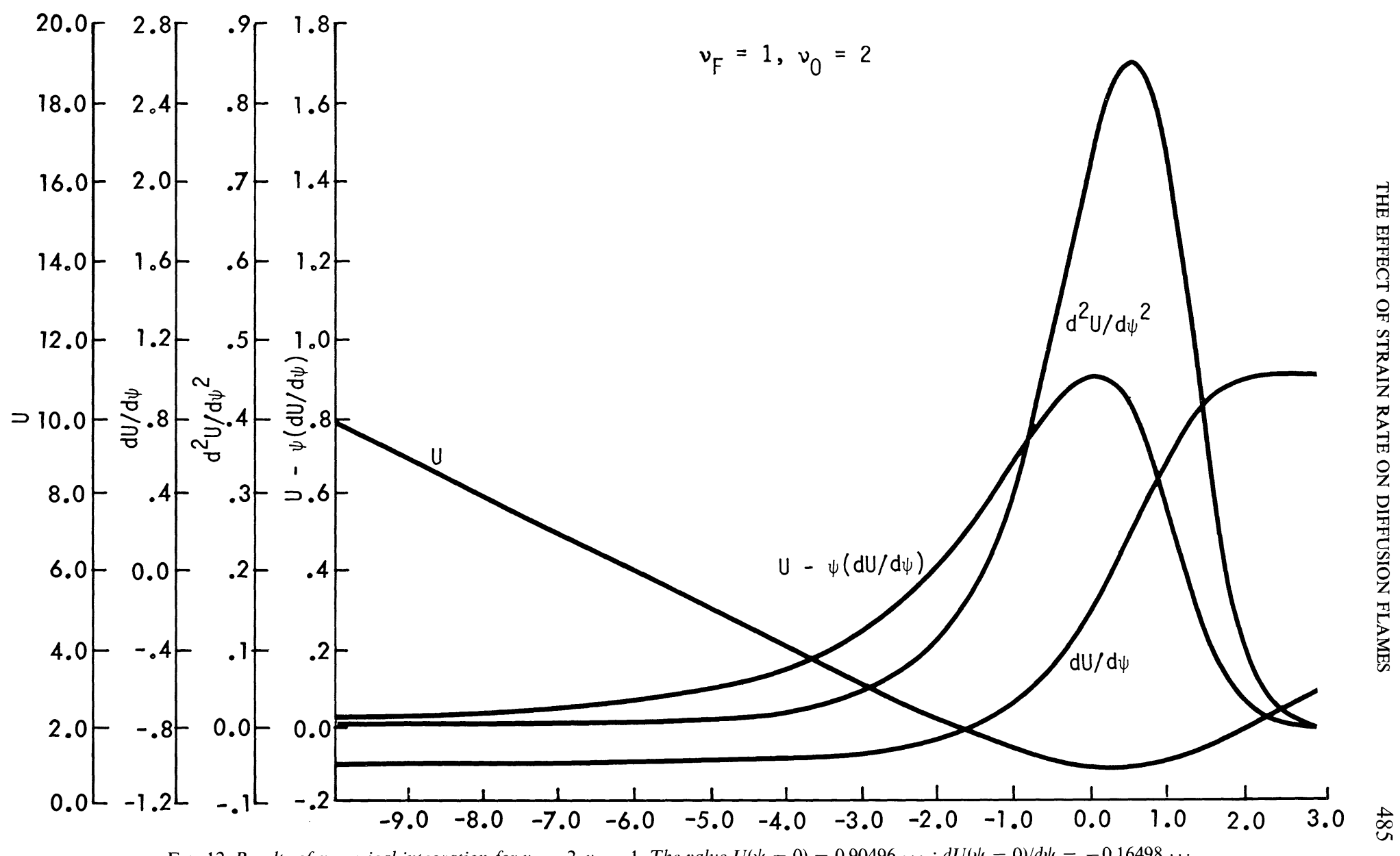

FIG. 12. Results of numerical integration for $v_{0}=2, v_{F}=1$. The value $U(\psi=0)=0.90496 \cdots ; d U(\psi=0) / d \psi=-0.16498 \cdots$ 


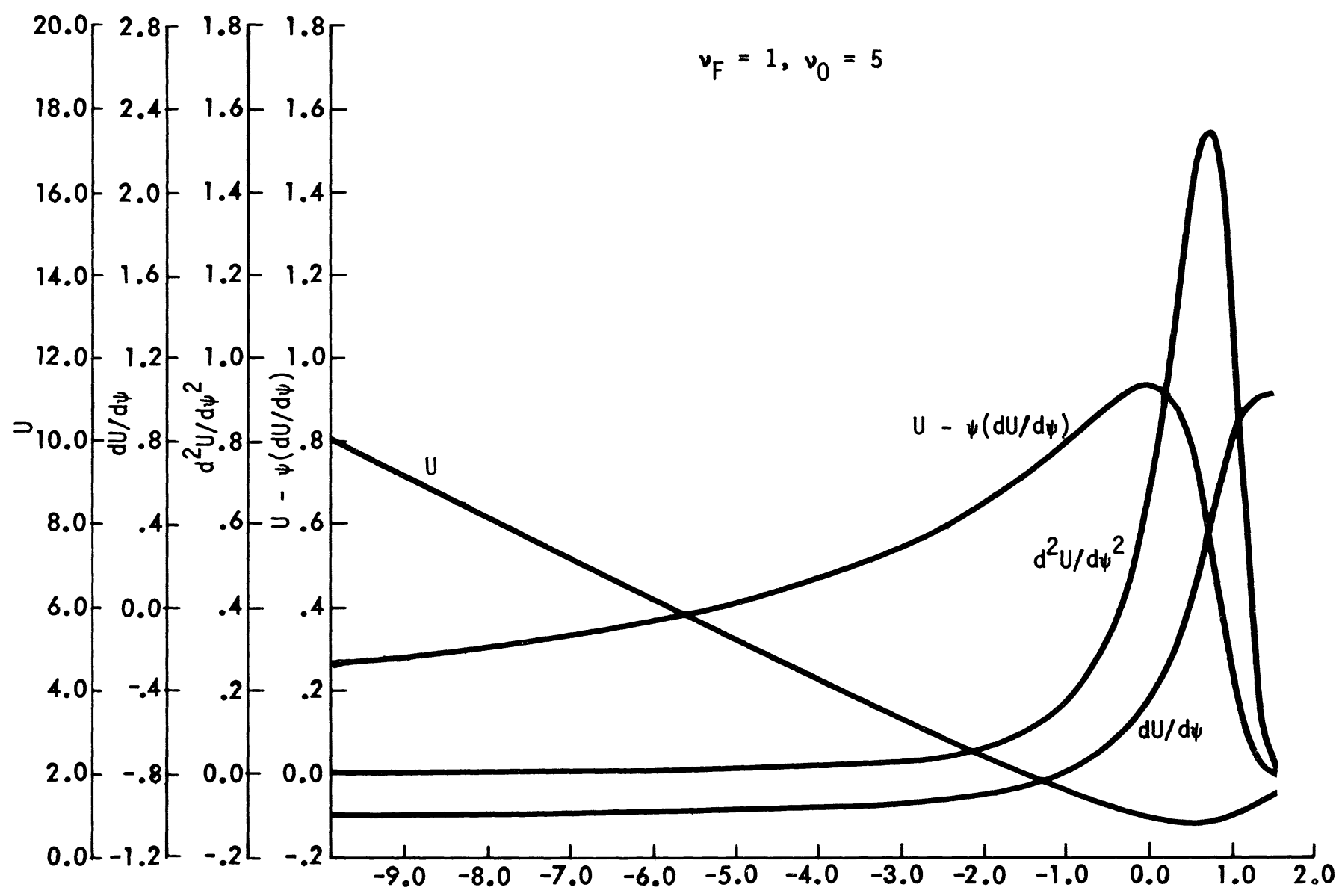


here

(A.24d)

$$
e_{0}=\left[\frac{\left(v_{0}+2\right)\left(v_{0}+v_{F}+1\right)}{\left(v_{F}-1\right)^{2}\left(c_{0}-a_{0}\right)^{v_{0}}}\right]^{1 /\left(v_{F}-1\right)}
$$

As $\chi \rightarrow-\infty$,

$$
\begin{gathered}
h \sim h_{*}-\delta^{b}\left\{c_{0}(-\chi)+e_{1}(-\chi)^{-\left(v_{F}+2\right) /\left(v_{0}-1\right)}\right. \\
\left.+O\left[(-\chi)^{-\left(3+2 v_{F}+v_{0}\right) /\left(v_{0}-1\right)}\right]\right\}+O\left(\delta^{2 b}\right) \\
F \sim \delta^{b}\left\{\left(c_{0}-a_{0}\right)(-\chi)+e_{1}(-\chi)^{-\left(v_{F}+2\right) /\left(v_{0}-1\right)}\right. \\
\left.+O\left[(-\chi)^{-\left(3+2 v_{F}+v_{0}\right) /\left(v_{0}-1\right)}\right]\right\}+O\left(\delta^{2 b}\right) \\
Y \sim \delta^{b}\left\{e_{1}(-\chi)^{-\left(v_{F}+2\right) /\left(v_{0}-1\right)}\right. \\
\left.+O\left[(-\chi)^{-\left(3+2 v_{F}+v_{0}\right) /\left(v_{0}-1\right)}\right]\right\}+O\left(\delta^{2 b}\right)
\end{gathered}
$$

here

$$
e_{1}=\left[\frac{\left(v_{F}+2\right)\left(v_{0}+v_{F}+1\right)}{\left(v_{0}-1\right)^{2}\left(c_{0}-a_{0}\right)^{v_{F}}}\right]^{1 /\left(v_{0}-1\right)}
$$

A.2. The reaction zone $\left(v_{0}>1\right.$ and/or $\left.v_{F}>1\right)$. First, the special case $v_{0}$ $=v_{F}=\beta<1$, in which $U$ is an even function of $\psi$, is considered. It is anticipated that $U=0$ for $\psi \geqq \psi_{0}$ and $\psi \leqq\left(-\psi_{0}\right)$, where $\psi_{0}$ is a finite point (to be determined) [17]; it is further anticipated that only a finite number of derivatives of the solution will be continuous at $\psi_{0}$. At least two derivatives will be continuous in the solution presented here, and that suffices for the second order boundary value problem posed by (A.14). If

$$
\mathscr{V}(\phi)=U(\psi) / \psi_{0}, \quad \phi=\psi / \psi_{0},
$$

then the eigenvalue $\lambda$ is introduced in (A.14a):

$$
\frac{d^{2} V}{d \phi^{2}}=\lambda\left(\mathscr{V}^{2}-\phi^{2}\right)^{\beta}, \quad \lambda=\psi_{0}^{2 \beta+1}, \quad 0<\beta<1,
$$

and (A.14b) becomes

$$
\phi=1: \mathscr{V}=\phi ; \quad \phi=0: \frac{d V}{d \phi}=0 .
$$

Preliminary trials suggest seeking a solution for $\phi \rightarrow 1$ in the form

$$
\mathscr{V}-\phi=A_{0}(1-\phi)^{2 /(1-\beta)}\left[1+\Delta(1-\phi)+\Gamma(1-\phi)^{2}+\cdots\right],
$$

where $A_{0}, \Delta, \Gamma, \cdots$ are constants to be determined. Hence,

$$
\left(\mathscr{V}^{2}-\phi^{2}\right)=(\mathscr{V}-\phi)^{\beta}(\mathscr{V}+\phi)^{\beta}=\left(2 A_{0}\right)^{\beta} \phi^{\beta}(1-\phi)^{2 \beta /(1-\beta)}
$$

$$
\begin{aligned}
& \left\{\left[1+\Delta(1-\phi)+\Gamma(1-\phi)^{2}+\cdots\right]\right. \\
& \left.\cdot\left[1+\frac{A_{0}(1-\phi)^{2 /(1-\beta)}}{2 \phi}+\cdots\right]\right\}^{\beta} .
\end{aligned}
$$


The number of continuous derivatives at $\phi=1$ compatible with (A.28) is $N$, the largest integer compatible with the inequality $1>\beta>[1-(2 / N)] \geqq 0$. Also, if $[2 /(1-\beta)]$ is a rational fraction, (A.28) continues as a power series; but if $[2 /(1-\beta)]$ is not a rational fraction, from (A.29) one sees that (A.28) continues as a series of ascending integral powers of $(1-\phi)$ only as long as $(1-\phi)^{n} \geqq(1$ $-\phi)^{2 /(1-\beta)}$, i.e., as long as $1>\beta>[1-(2 / n)] \geqq 0$ - the inequality just givenwhere $n$ is an integer. In any case, as long as $\beta>0$, (A.28) remains intact through the quadratic terms given, and to this order of approximation one can drop the $\left[A_{0}(1-\phi)^{2 /(1-\beta)} / 2 \phi\right]$ term in (A.29). Forming $\left(d^{2} V / d \phi^{2}\right)$ from (A.28) and substituting it, together with (A.29), in (A.27a) and equating coefficients of equal powers, one obtains

$$
\lambda=\psi_{0}^{2 \beta+1}=\left(2 A_{0}\right)^{1-\beta}(1+\beta) /(1-\beta)^{2}, \quad \Delta=-\frac{\beta(1+\beta)}{(1-\beta)(3+\beta)}
$$

(A.31) $\Gamma=-\frac{\beta(1+\beta)\left(9-12 \beta-7 \beta^{2}-2 \beta^{3}\right)}{12(1-\beta)^{2}(3+\beta)^{2}}$.

The solution is complete upon determination of $A_{0}$. (If $A_{0}=O(1)$, or $A_{0}=O(1-\beta)$ as in (A.32) below, or almost anything nonexponential, $\left.\lambda(\beta \rightarrow 1) \sim(1-\beta)^{-2} \rightarrow \infty\right)$. One possibility for estimating $A_{0}$ is to postulate the validity of (A.28) over the entire domain $0 \leqq \phi \leqq 1$, rather than just in the region $\phi \rightarrow 1$; (A.27b) requires that $d V / d \phi=0$ at $\phi=0$, which implies

$$
A_{0}=\frac{1-\beta}{2+(3-\beta) \Delta+2(2-\beta) \Gamma}
$$

for $\beta=1 / 3, \Delta=-1 / 5$ and $\Gamma=-7 / 225$, so

$$
A_{0}=\frac{1}{3-\frac{4}{5}-\frac{7}{45}}
$$

this suggests about a seven percent error owing to series truncation. The error is about twenty percent at $\beta=1 / 2$. An alternate procedure, aimed at a slightly more accurate approximation at small $\phi$, retains (A.32) to enforce the symmetry condition at $\phi=0$, and (A.30), so the coefficients of the first two terms (only) in the representation (A.28) are assigned on the basis of the correct series expansion as $\phi \rightarrow 1$. Instead of similarly assigning the third coefficient as well, collocation at $\phi=0$ is used; substituting (A.28) in (A.27a) and requiring compatibility at $\phi=0$ gives

$$
A_{0}=\frac{2}{(1+\Delta+\Gamma)^{2}}\left\{\frac{(1+\beta)+(3-\beta) \Delta+(3-\beta)(2-\beta) \Gamma}{1+\beta}\right\}^{1 / \beta}
$$

Equations (A.32) and (A.33) now give two equations for two unknowns $A_{0}$ and $\Gamma$ and thus, with (A.30), complete determination of the solution (A.28). In fact, (A.31) furnishes an initial estimate of $\Gamma$ for iteration.

More generally, if $v_{F}<1, v_{0}$ arbitrary, one anticipates that $U=\psi$ at a finite positive value of $\psi$ (denoted $\psi_{0}$ ), but in general $U \rightarrow-\psi$ only as $(-\psi) \rightarrow \infty$. (If $v_{0}<1, U \rightarrow-\infty$ at a finite negative value of $\psi$, different in magnitude from $\psi_{0}$, 
except when $v_{0}=v_{F}=\beta$, just treated.) Under (A.26), (A.14) now becomes

$$
\frac{d^{2} V}{d \phi^{2}}=\lambda(\mathscr{V}-\phi)^{v_{F}}(\mathscr{V}+\phi)^{v_{0}}, \quad \lambda=\psi_{0}^{v_{0}+v_{F}+1},
$$

and one seeks a solution for $\phi \rightarrow 1$ in the form

$$
\mathscr{V}-\phi=A_{0}(1-\phi)^{2 /\left(1-v_{F}\right)}\left[1+\Delta(1-\phi)+\Gamma(1-\phi)^{2}+\cdots\right] .
$$

Substituting and equating powers yields

$$
\begin{aligned}
& \lambda=\psi_{0}^{v_{0}+v_{F}+1}=2^{1-v_{0}} A_{0}^{1-v_{F}}\left(1+v_{F}\right) /\left(1-v_{F}\right)^{2} \\
& \Delta=-\frac{v_{0}\left(1+v_{F}\right)}{\left(3+v_{F}\right)\left(1-v_{F}\right)} \\
& \Gamma=-\frac{v_{0}\left(1+v_{F}\right)\left[9-3 v_{F}-9 v_{0}-2 v_{0} v_{F}-5 v_{F}^{2}-v_{F}^{2}\left(v_{0}+v_{F}\right)\right]}{12\left(1-v_{F}\right)^{2}\left(3+v_{F}\right)^{2}}
\end{aligned}
$$

If $v_{0} \geqq 1$, an estimate of $A_{0}$ permits one to initiate integration in the direction of decreasing $\phi$ from $\phi=1$ (and implicitly from a trial value of $\psi_{0}$ ). Conventional iteration procedures should readily yield the $A_{0}$ compatible with $U \rightarrow-\psi$ as $\psi \rightarrow-\infty$. If $v_{0}<1$, then a solution for $\left(-\psi_{1}\right) \geqq(-\psi)>0$, where the positive quantity $\left(-\psi_{1}\right)$ is the analogue of $\psi_{0}$, is furnished by

$$
\frac{d^{2} \overline{\mathscr{V}}}{d \bar{\phi}^{2}}=\bar{\lambda}(\overline{\mathscr{V}}-\bar{\phi})^{v_{0}}(\overline{\mathscr{V}}+\bar{\phi})^{v_{F}}, \quad \bar{\lambda}=\left(-\psi_{1}\right)^{v_{0}+v_{F}+1},
$$

where

$$
\overline{\mathscr{V}}=U /\left(-\psi_{1}\right), \quad \bar{\phi}=(-\psi) /\left(-\psi_{1}\right) .
$$

Thus, as $\bar{\phi} \rightarrow 1$,

$$
\begin{aligned}
\bar{V}-\bar{\phi} & =A_{1}(1-\bar{\phi})^{2 /\left(1-v_{0}\right)}\left[1+\Delta_{1}(1-\bar{\phi})+\Gamma_{1}(1-\bar{\phi})^{2}+\cdots\right] \\
\bar{\lambda} & =\left(-\psi_{1}\right)^{v_{0}+v_{F}+1}=2^{1-v_{F}} A_{1}^{1-v_{0}}\left(1+v_{0}\right) /\left(1-v_{0}\right)^{2} \\
\Delta_{1} & =-\frac{v_{F}\left(1+v_{0}\right)}{\left(3+v_{0}\right)\left(1-v_{0}\right)} \\
\Gamma_{1} & =-\frac{v_{F}\left(1+v_{0}\right)\left[9-3 v_{0}-9 v_{F}-2 v_{0} v_{F}-5 v_{0}^{2}-v_{0}^{2}\left(v_{0}+v_{F}\right)\right]}{12\left(1-v_{0}\right)^{2}\left(3+v_{0}\right)^{2}} .
\end{aligned}
$$

Continuity of $U$ and $d U / d \psi$ at $\psi=0$ furnishes two conditions for the two unknowns $A_{0}$ and $A_{1}$ (the domains of validity of the series representations of $\mathscr{V}$ and $\overline{\mathscr{V}}$ are being extended to $\psi=0$ ):

$$
\begin{aligned}
& -\psi_{1} A_{1}\left(1+\Delta_{1}+\Gamma_{1}\right)=\psi_{0} A_{0}(1+\Delta+\Gamma), \\
& \frac{A_{0}}{1-v_{F}}\left[2+\left(3-v_{F}\right) \Delta+2\left(2-v_{F}\right) \Gamma\right]
\end{aligned}
$$

$$
+\frac{A}{1-v_{0}}\left[2+\left(3-v_{0}\right) \Delta_{1}+2\left(2-v_{0}\right) \Gamma_{1}\right]=2 .
$$


Numerical results for cases with $v_{0}$ and $v_{F}$ less than unity, developed from the theory just presented in (A.26)-(A.44), are presented in Figs. 14-16. Because the methods fail for $\beta \rightarrow 1$, a more formal (but more widely applicable) approach based on a type of Picard iteration (rather than series truncation) is now developed. The authors are grateful to Prof. Bernard Budiansky of Harvard University for his contribution in the following approach.

Attention is limited to the case in which both stoichiometric coefficients are equal and less than unity; the boundary value problem is given by (A.27), where it is noted that $d \mathscr{V} / d \phi=0$ at $\phi=1$. By definition,

$$
w(z)=V^{2}-\phi^{2}, \quad z=1-\phi,
$$

where the present use of $w$ and $z$ is clearly distinguished from their use as the axial velocity component and axial coordinate in a cylindrical polar system introduced above (4.12b). By successive integrations of (A.27a), in view of (A.27b),

$$
\mathscr{V}=(1-z)+\lambda \int_{0}^{z} d \eta \int_{0}^{\eta}[w(\zeta)]^{\beta} d \zeta
$$

so

$$
\begin{aligned}
w(z)=(\mathscr{V}-\phi)(\mathscr{V}+\phi)= & \left\{2(1-z)+\lambda \int_{0}^{z} d \eta \int_{0}^{\eta}[w(\zeta)]^{\beta} d \zeta\right\} \\
& \cdot\left\{\lambda \int_{0}^{z} d \eta \int_{0}^{\eta}[w(\zeta)]^{\beta} d \zeta\right\}
\end{aligned}
$$

As $\phi \rightarrow 1$, from (A.28),

$$
\begin{aligned}
\mathscr{V} & =\phi+A_{0}(1-\phi)^{2 /(1-\beta)}[1+\cdots], \\
w & =2 A_{0} z^{2 /(1-\beta)}[1+\cdots] .
\end{aligned}
$$

Substituting (A.49) in (A.47) for $z \rightarrow 0$ recovers part of (A.30) :

$$
\bar{c}^{1-\beta}=\lambda(1-\beta)^{2} /(1+\beta), \quad \bar{c}=2 A_{0} .
$$

If

$$
w(z)=\bar{c} \bar{U}, \quad z=\bar{T}^{(1-\beta) /(1+\beta)} \equiv \bar{T}^{\bar{\alpha}},
$$

then (A.47) may be rewritten, by (A.50) and (A.51),

$$
\begin{aligned}
\bar{U}= & \left\{2\left(1-\bar{T}^{\alpha}\right)+\frac{\bar{c}}{1+\beta} \int_{0}^{\bar{T}} \tau^{-\gamma} d \tau \int_{0}^{\tau} \sigma^{-\gamma}[\bar{U}(\sigma)]^{\beta} d \sigma\right\} \\
& \cdot\left\{\frac{1}{1+\beta} \int_{0}^{\bar{T}} \tau^{-\gamma} d \tau \int_{0}^{\tau} \sigma^{-\gamma}[\bar{U}(\sigma)]^{\beta} d \sigma\right\},
\end{aligned}
$$

where $\gamma=2 \beta /(1+\beta)$. 

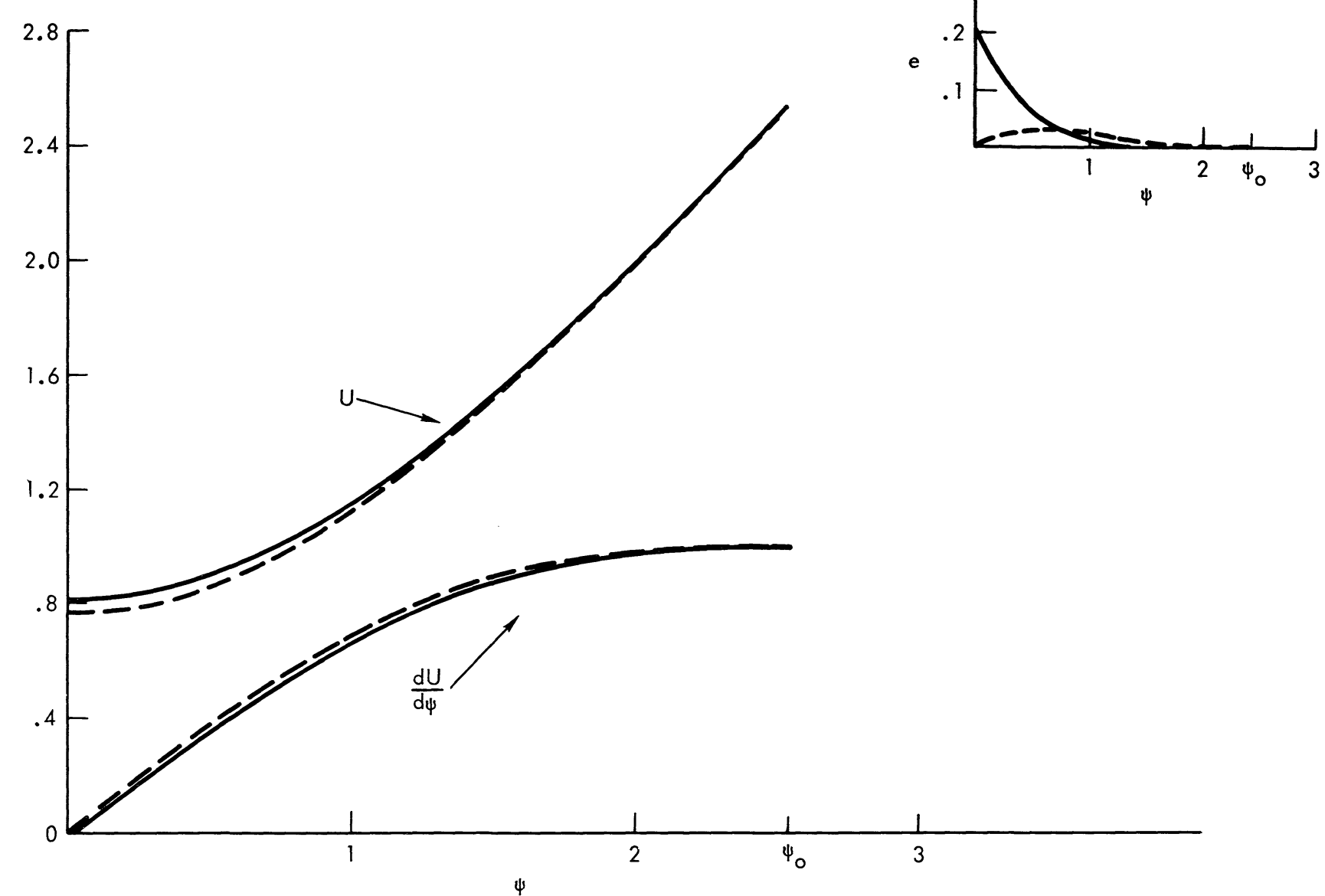

FIG. 14. Approximate analytic solution to the boundary value problem posed by (A.26)-(A.27) for $\beta=0.5$ by assignment of constants in the form (A .28). The dashed lines denote the solution with constants assigned according to (A.30)-(A.32); the solid lines, constants assigned according to (A.30), (A .32), and (A.33). The insert gives the error (ratio of the local residual to the largest of the two terms of the differential equation (A.29a)), and demonstrates the overall superiority of the solid-line solution, for which $U(\psi=0) \doteq 0.7602, d U(\psi=0) / d \psi=0$, and $\psi_{0} \doteq 2.487$. For $\beta \rightarrow 0$, the two methods of assignment of constants are both quite accurate, while as $\beta$ increases the solid-line solution becomes markedly superior. 


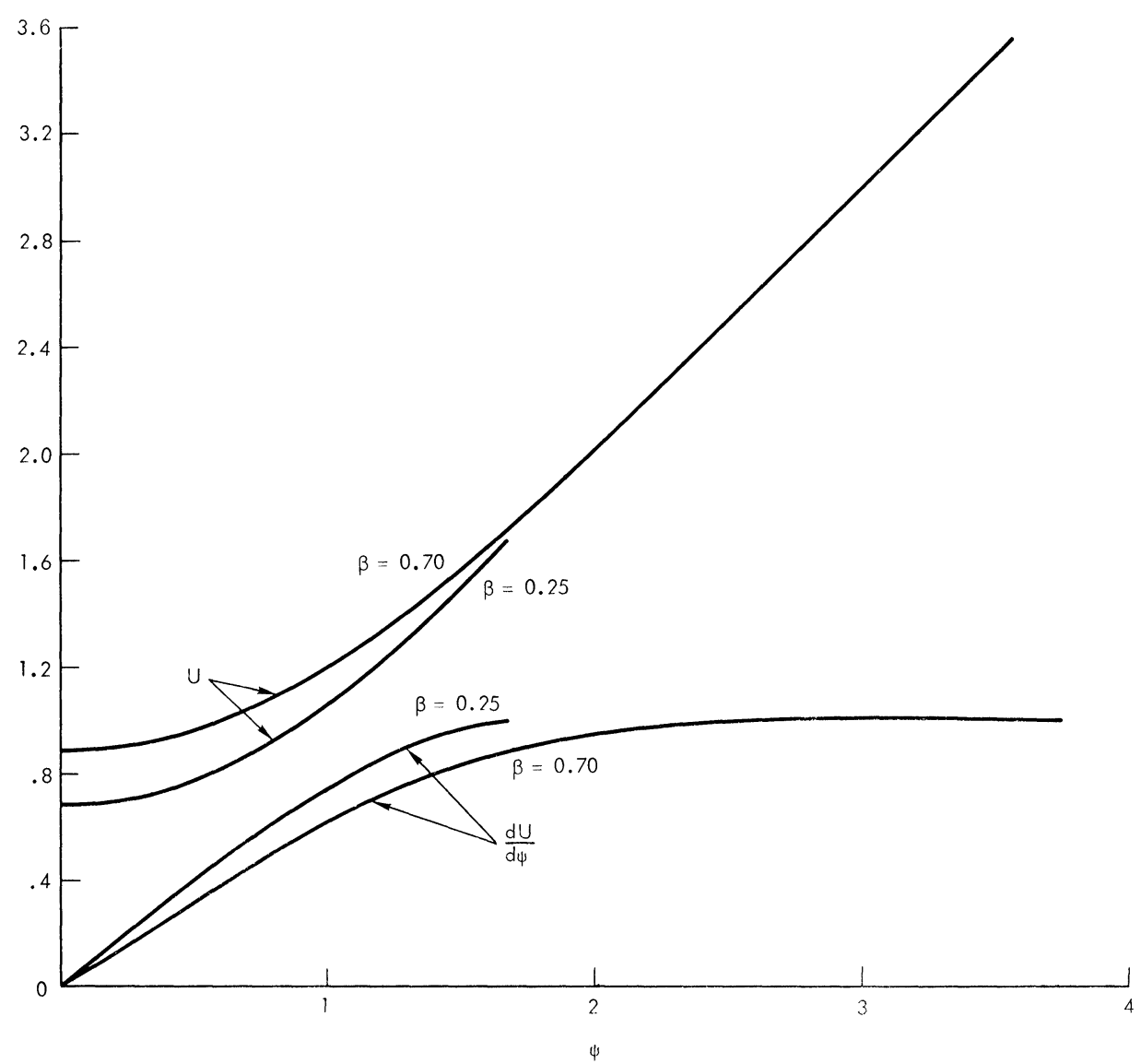

FIG. 15. Approximate analytic solution to the boundary value problem posed by (A.26)-(A.27), via (A.28), with constants assigned by (A.30), (A.32), and (A.33). For $\beta=0.25, U(\psi=0) \doteq 0.6712, d U(\psi$ $=0) / d \psi=0, \psi_{0} \doteq 1.659 ;$ for $\beta=0.70, U(\psi=0) \doteq 0.8073, d U(\psi=0) / d \psi=0, \psi_{0} \doteq 3.693$. For $\beta \geqq 0.75$, the method of assigning constants in (A.28) must be modified because of singularities that arise.

From (A.27), (A.45), and (A.51),

$$
1=\lambda \int_{0}^{1}\left(\mathscr{V}^{2}-\phi^{2}\right)^{\beta} d \phi=\lambda \int_{0}^{1}[w(z)]^{\beta} d z=\lambda \bar{c}^{\beta} \int_{0}^{1}[\bar{U}(z)]^{\beta} d z
$$

$$
=\lambda \bar{c}^{\beta} \bar{\alpha} \int_{0}^{1} t^{-\gamma}[\bar{U}(t)]^{\beta} d t
$$

From (A.50),

$$
\bar{D} \equiv \frac{\bar{c}}{1-\beta}=\left\{\int_{0}^{1} t^{-\gamma}[\bar{U}(t)]^{\beta} d t\right\}^{-1}
$$




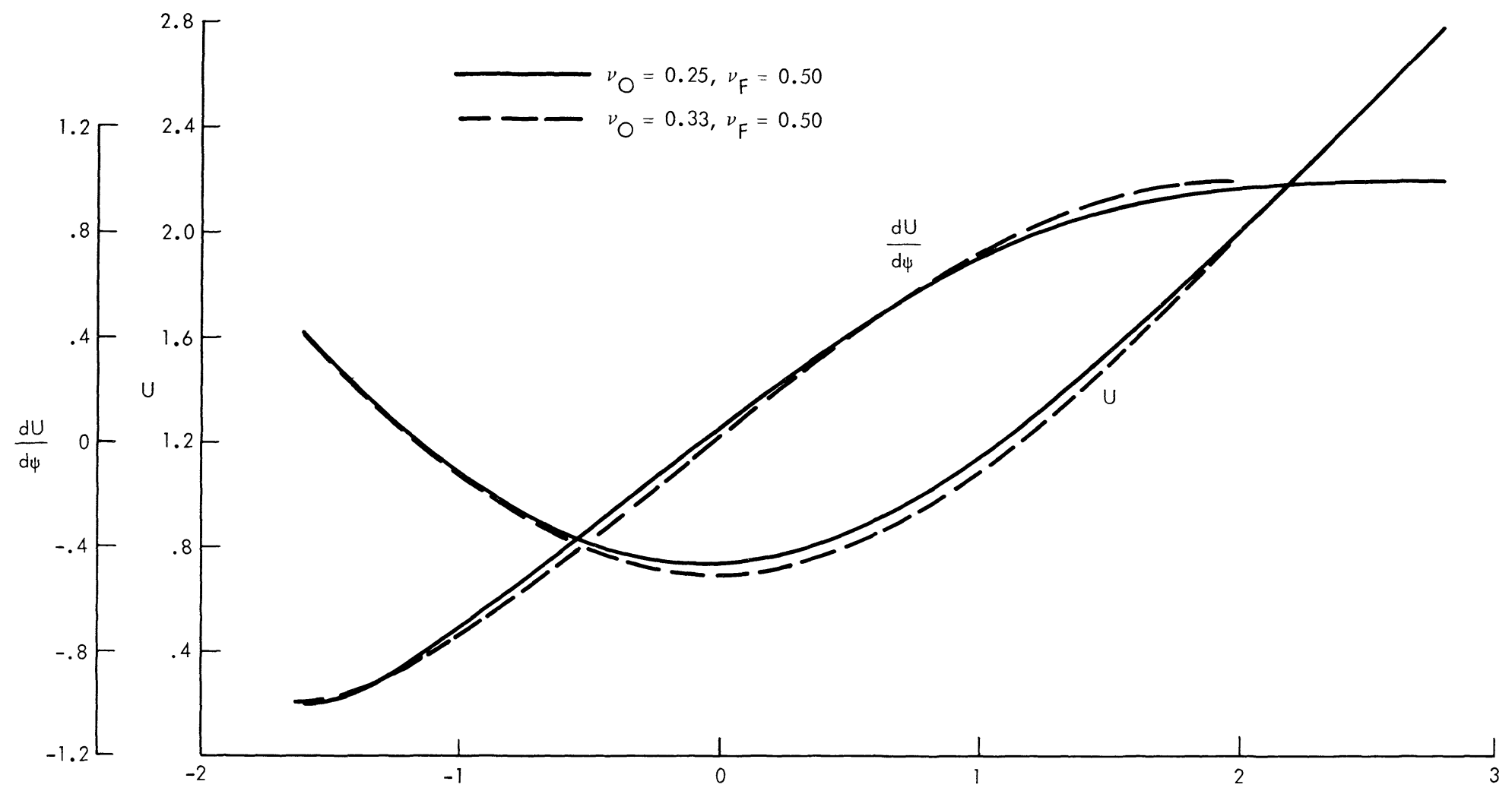

FIG. 16. Approximate analytic solution to the boundary value problem posed by (A.27) subject to the boundary conditions $U\left(\psi \geqq \psi \psi_{0}\right)=\psi, U\left(\psi \leqq \psi\left(\psi_{1}\right)=-\psi\right.$, where $\psi_{0}>0, \psi_{1}<0$. Here solutions found by (A.34)-(A.44) are given for $v_{0}=0.25, v_{F}=0.50\left(\right.$ for which $U(\psi=0) \doteq 0.7425, d U(\psi=0) / d \psi \doteq 0.0307, \psi_{0} \doteq 2.788, \psi$ $\doteq-1.598)$, and for $v_{0}=0.25, v_{F}=0.33$ (for which $\left.U(\psi=0) \doteq 0.6997, d U(\psi=0) / d \psi \doteq 0.0156, \psi_{0} \doteq 1.945, \psi_{1} \doteq-1.645\right)$. The method of solution used here is accurate only for $v_{0}, v_{F} \leqq 0.5$. 
From (A.54), (A.52) is

$$
\begin{aligned}
\bar{U}(\bar{T})= & \left\{2\left(1-\bar{T}^{\bar{\alpha}}\right)+\bar{\alpha} \bar{D} \int_{0}^{\bar{T}} \tau^{-\gamma} d \tau \int_{0}^{\tau} \sigma^{-\gamma}[\bar{U}(\sigma)]^{\beta} d \sigma\right\} \\
& \cdot\left\{\int_{0}^{\bar{T}} \tau^{-\gamma} d \tau \int_{0}^{\tau} \tau^{-\gamma}[\bar{U}(\sigma)]^{\beta} d \sigma\right\} /(1+\beta) .
\end{aligned}
$$

Since $\bar{U}(\bar{T} \rightarrow 0) \sim \bar{T}^{2 /(1+\beta)}$, as $\bar{T} \rightarrow 0$ it holds that $\bar{T}^{-\gamma} \bar{U}^{\beta} \rightarrow 1$ and thus the integrands are bounded.

The form (A.55) is suitable for iteration : a trial for $\bar{U}$ substituted into the righthand side yields a new approximation; convergence may be suggested by suitable invariance of $D$ under successive approximations to $\bar{U}$. The solution is given explicitly by

(A.56) $\mathscr{V}(\phi ; \beta)=\left\{\bar{D}(1-\beta) \bar{U}(\bar{T})+\phi^{2}\right\}^{1 / 2}, \quad \bar{T}=(1-\phi)^{1 / \bar{\alpha}}, \quad \bar{\alpha}=\frac{1-\beta}{1+\beta}$,

(A.57) $\lambda=\frac{\bar{D}^{1-\beta}(1+\beta)}{(1-\beta)^{1+\beta}}$.

To select an initial guess for $\bar{U}$, one may note that for $\beta=0$,

$$
V=\left(1+\phi^{2}\right) / 2 \Rightarrow w=\bar{c} \bar{U}=\bar{U}=\bar{T}^{2}(1-\bar{T} / 2)^{2} \text {. }
$$

For $\beta>0$, a straightforward generalization of (A.58) for the initial trial is

$$
\bar{U}=\bar{c}^{-1} \bar{T}^{2 /(1+\beta)}(1-\bar{T} / 2)^{2 /(1+\beta)},
$$

where $\bar{c}$ may be taken as unity or may be approximated by (A.54). Computed values of $\lambda$ for several $\beta$ are given in Table 2 .

TABLE 2

Eigenvalues for the flame zone boundary value problem as a function of the stoichiometric coefficient

\begin{tabular}{ll|cc|cc}
\hline$\beta$ & \multicolumn{1}{c|}{$\lambda$} & $\beta$ & $\lambda$ & $\beta$ & $\lambda$ \\
\hline 0. & 1. & 0.35 & 3.15155 & 0.70 & 23.2374 \\
0.05 & 1.14384 & 0.40 & 3.91305 & 0.75 & 35.2065 \\
0.10 & 1.31888 & 0.45 & 4.94920 & 0.80 & 56.8135 \\
0.15 & 1.53439 & 0.50 & 6.39134 & 0.85 & 101.688 \\
0.20 & 1.80316 & 0.55 & 8.44902 & 0.90 & 223.402 \\
0.25 & 2.14318 & 0.60 & 11.4672 & 0.95 & 846.161 \\
0.30 & 2.58016 & 0.65 & 16.0377 & 0.99 & 20047.3 \\
\hline
\end{tabular}

A.3. The reaction zone: higher order terms. The next order linear boundary value problem retains convective transport (absent to lowest order of approximation) as a forcing function. Substitution of (A.5) in (A.1) and (A.2) gives, in view of (A.9)-(A.11), for any $v_{0}, v_{F}$,

(A.60) $\frac{d^{2}}{d \chi^{2}}\left(\mathscr{F}_{1}-\mathscr{H}_{1}\right)+2 \zeta_{*} \frac{d}{d \chi}\left(\mathscr{F}_{0}-\mathscr{H}_{0}\right)=0 \Rightarrow \mathscr{F}_{1}-\mathscr{H}_{1}=-2 \zeta_{*} a_{0}\left(\zeta^{2} / 2\right)$, 
(A.61)

$$
\frac{d^{2}}{d \chi^{2}}\left(\mathscr{Y}_{1}-\mathscr{H}_{1}\right)+2 \zeta_{*} \frac{d}{d \chi^{2}}\left(\mathscr{Y}_{0}-\mathscr{H}_{0}\right)=0 \Rightarrow \mathscr{Y}_{1}-\mathscr{H}_{1}=-2 \zeta_{*} c_{0}\left(\zeta^{2} / 2\right)
$$

$$
\frac{d^{2} \mathscr{H}_{1}}{d \chi^{2}}+2 \zeta_{*} \frac{d \mathscr{H}_{0}}{d \chi}=\frac{d^{2} \mathscr{H}_{0}}{d \chi^{2}}\left[\frac{v_{F} \mathscr{F}_{1}}{\mathscr{F}_{0}}+\frac{v_{0} \mathscr{Y}_{1}}{\mathscr{Y}_{0}}-\frac{\theta \mathscr{H}_{0}}{\left(h_{*}+\alpha\right)^{2}}\right]
$$

The constants of integration multiplying the complementary functions in the Shvab-Zeldovich integrals have again been set to zero by comparing the asymptotic expansions with the exact integrals (3.1)-(3.3). Clearly $\theta$ enters independently of $\delta$ to this order of approximation.

As $\chi \rightarrow \infty$ the asymptotic behavior is, with the aid of the lowest order solution for the reaction zone, for $v_{0}$ arbitrary and $v_{F}>1$ :

$$
\begin{aligned}
\mathscr{H}_{1} & \sim-2 \zeta_{*}\left(-a_{0}\right) \chi^{2} / 2+e_{2} \chi^{-v_{1}+2}+\cdots, \\
\mathscr{Y}_{1} & \sim-2 \zeta_{*}\left(c_{0}-a_{0}\right) \chi^{2} / 2+e_{2} \chi^{-v_{1}+2}+\cdots, \\
\mathscr{F}_{1} & \sim e_{2} \chi^{-v_{1}+2}+\cdots,
\end{aligned}
$$

where $e_{2}$ is of order unity and independent of $\chi$, and

$$
v_{1}=\frac{v_{0}+v_{F}+1}{v_{F}-1} \text {. }
$$

The terms involving $e_{2}$ would be exponentially small if $v_{F}=1$, and precisely zero if $v_{F}<1$. Analogous statements, mutatis mutandis, hold as $\chi \rightarrow-\infty$.

A.4. The fuel and oxidant zones: higher order terms $\left(v_{0}>1, v_{F}>1\right)$. Substitution of (A.3) and (A.4) and (A.2) yields in view of the boundary conditions, of the known exact Shvab-Zeldovich integrals (3.1)-(3.3) valid for any $\delta$, and of (A.7) and (A.8):

$$
\begin{array}{cc}
F_{1}(\zeta)+H_{1}(\zeta)=0, & Y_{1}(\zeta)+H_{1}(\zeta)=0, \\
f_{1}(\zeta)+h_{1}(\zeta)=0, & y_{1}(\zeta)+h_{1}(\zeta)=0
\end{array}
$$

the upstream terms vanish as $\zeta \rightarrow \infty$; the downstream, as $\zeta \rightarrow-\infty$. Supplementing these integrals in the upstream oxidant zone is

$$
L_{1}\left(F_{1}\right)=\left(\frac{d^{2}}{d \zeta^{2}}+2 \zeta \frac{d}{d \zeta}\right) F_{1}=F_{1}^{v_{F}} Y_{0}^{v_{0}} \exp \left[-\frac{\theta\left(h_{*}-H_{0}\right)}{\left(h_{*}+\alpha\right)\left(H_{0}+\alpha\right)}\right]
$$

In the downstream fuel zone,

$$
L\left(y_{1}\right)=\left(\frac{d^{2}}{d \zeta^{2}}+2 \zeta \frac{d}{d \zeta}\right) y_{1}=y_{1}^{v_{0}} f_{0}^{v_{F}} \exp \left[-\frac{\theta\left(h_{*}-h_{0}\right)}{\left(h_{*}+\alpha\right)\left(h_{0}+\alpha\right)}\right] .
$$

Substituting from (A.7), (A.8), and (A.12) gives

$$
\begin{gathered}
F_{1}\left(\zeta \rightarrow \zeta_{*}\right)=A_{1}\left(\zeta-\zeta_{*}\right)^{-v_{1}}+B_{1}\left(\zeta-\zeta_{*}\right)^{-v_{1}+1}+\cdots \\
y_{1}\left(\zeta \rightarrow \zeta_{*}\right)=A_{2}\left(\zeta_{*}-\zeta\right)^{-v_{2}}+B_{2}\left(\zeta_{*}-\zeta\right)^{-v_{2}+1}+\cdots
\end{gathered}
$$


Here

$$
\begin{aligned}
& A_{1}=\left[\frac{\left(2+v_{0}\right)\left(1+v_{0}+v_{F}\right)}{\left(v_{F}-1\right)^{2}\left(c_{0}-a_{0}\right)^{v_{0}}}\right]^{1 /\left(v_{F}-1\right)} \\
& A_{2}=\left[\frac{\left(2+v_{F}\right)\left(1+v_{0}+v_{F}\right)}{\left(v_{0}-1\right)^{2}\left(c_{0}-a_{0}\right)^{v_{F}}}\right]^{1 /\left(v_{0}-1\right)}
\end{aligned}
$$

From the definitions of $e_{0}$ and $e_{1}$, the first terms in the expansions for $F_{1}$ and $y_{1}$ near $\zeta_{*}$ match with appropriate terms in the asymptotic behavior of the lowest order reaction-zone solution as $\chi \rightarrow \pm \infty$. Thus, well-posed boundary value problems for the next-to-lowest-order fuel and oxidant zone solutions have been presented. When $v_{F}>1$, sufficient fuel penetrates the reaction zone for corrections of algebraic order of smallness (in the inverse of the first Damköhler number) to the lowest order (order unity) solution for the oxidant zone. Hence, finite-rate reaction occurs to higher orders of approximation in the oxidant zone, in which no reaction occurred to lowest order, because to lowest order one reactant was absent. Analogous statements may be made for the fuel zone.

Appendix B. The time-dependent near-equilibrium irreversible limit. If 1 $\gg D_{1}^{-1}>0$, as opposed to the case $D_{1} \rightarrow \infty$ treated in $\S 4$, then the boundary value problem posed by (2.4)-(2.5) and the initial boundary conditions stated in $\S 2$ must be studied. Attention will be confined to the case of a single flame. Equation (4.6) presents exact solutions to (2.5) valid for any $D_{1}$ under the boundary initial conditions of interest here; any local asymptotic series expansions (for $1 \gg D_{1}^{-1}$ $>0$, say) for $Y, F$, and $h$ must be compatible with (4.6). As in the steady case treated in Appendix A, there is a three-region subdivision of the flow : an upstream oxidant region and a downstream fuel region, both of which are time-dependent to lowest order of approximation, and a relatively thin intermediate reaction zone, which is quasi-steady to lowest order (only) in that time derivatives are not present. Because time enters parametrically (only) in the flame zone to lowest order, the results developed previously for the steady case serve as a guide.

For the boundary initial conditions of interest here the enthalpy at the thin flame is constant in time at the value $h_{*}$ given by (3.5). However, a more general formulation of the boundary conditions (for example) could yield $h_{*}$ varying in time, and this more general case will be adopted in the formal presentation. Hence, incorporation of the Arrhenius factor in the expansion parameter (as was done in (A.6) for the steady case) is not adopted; the expansion parameter is $\bar{\delta}=D_{1}^{-1}$, $1 \gg \bar{\delta}>0$.

Upstream oxidant zone:

$$
\begin{aligned}
& F(\xi, t ; \bar{\delta})=o(1) \\
& Y(\xi, t ; \bar{\delta})=Y_{0}(\xi, t)+o(1) \\
& h(\xi, t ; \bar{\delta})=H_{0}(\xi, t)+o(1) .
\end{aligned}
$$


Downstream fuel zone:

$$
\begin{aligned}
& F(\xi, t ; \bar{\delta})=f_{0}(\xi, t)+o(1) \\
& Y(\xi, t ; \bar{\delta})=o(1) \\
& h(\xi, t ; \bar{\delta})=h_{0}(\xi, t)+o(1) .
\end{aligned}
$$

Intermediate reaction zone $\left[\chi=\left(\xi-\xi_{*}(t)\right) / \bar{\delta}^{b}, b=1 /\left(v_{0}+v_{F}+1\right)\right]$ :

$$
\begin{array}{ll}
F(\xi, t ; \bar{\delta})= & \bar{\delta}^{b} \mathscr{F}_{0}(\chi, t)+\bar{\delta}^{2 b} \mathscr{F}_{1}(\chi, t)+\cdots ; \\
Y(\xi, t ; \bar{\delta})= & \bar{\delta}^{b} \mathscr{Y}_{0}(\chi, t)+\bar{\delta}^{2 b} \mathscr{Y}_{1}(\chi, t)+\cdots ; \\
h(\xi, t ; \bar{\delta})=h_{*}(t)- & \bar{\delta}^{b} \mathscr{H}_{0}(\chi, t)-\bar{\delta}^{2 b} \mathscr{H}_{1}(\chi, t)-\cdots
\end{array}
$$

Analogous to the steady case, substitution of (B.1) and (B.2) in (2.4) and (2.5) may be anticipated to recover, to lowest order of approximation, the so-called Burke-Schumann solution given in (4.6):

$$
\begin{aligned}
& Y_{0}+H_{0}=c_{1} \operatorname{erf}\left[\frac{\xi-m(t)}{r(t)}\right]+c_{2}, \quad H_{0}=c_{3} \operatorname{erf}\left[\frac{\xi-m(t)}{r(t)}\right]+c_{4} \\
& h_{0}=c_{1} \operatorname{erf}\left[\frac{\xi-m(t)}{r(t)}\right]+c_{2}, \quad f_{0}+h_{0}=c_{3} \operatorname{erf}\left[\frac{\xi-m(t)}{r(t)}\right]+c_{4}
\end{aligned}
$$

To lowest order of approximation in the reaction zone,

$$
\begin{aligned}
& \frac{\partial^{2}}{\partial \eta^{2}}\left(\mathscr{F}_{0}-\mathscr{H}_{0}\right)=0 \Rightarrow \mathscr{F}_{0}-\mathscr{H}_{0}=\bar{a}_{0}(\tau) \eta, \\
& \frac{\partial^{2}}{\partial \eta^{2}}\left(\mathscr{Y}_{0}-\mathscr{H}_{0}\right)=0 \Rightarrow \mathscr{Y}_{0}-\mathscr{H}_{0}=\bar{c}_{0}(\tau) \eta, \\
& \frac{\partial^{2} \mathscr{H}_{0}}{\partial \eta^{2}}=\mathscr{F}_{0}^{v_{F}} \mathscr{Y}_{0}^{v_{0}}, \quad \eta=\left\{\exp \left[-\theta /\left(h_{*}(t)+\alpha\right)\right]\right\}^{1 / 2} \chi .
\end{aligned}
$$

Matching yields for the case of interest here, implied by (B.4) and (B.5), in which $h_{*}$ is constant,

$$
\begin{aligned}
& \bar{a}_{0}\left\{\exp \left[-\theta /\left(h_{*}+\alpha\right)\right]\right\}^{1 / 2}=\frac{2 c_{3}}{\pi^{1 / 2} r(t)} \exp \left\{-\left[\frac{\xi_{*}(t)-m(t)}{r(t)}\right]^{2}\right\}, \\
& \bar{c}_{0}\left\{\exp \left[-\theta /\left(h_{*}+\alpha\right)\right]\right\}^{1 / 2}=\frac{2 c_{1}}{\pi^{1 / 2} r(t)} \exp \left\{-\left[\frac{\xi_{*}(t)-m(t)}{r(t)}\right]^{2}\right\} .
\end{aligned}
$$

By the transformation given in (A.13), with $a_{0} \rightarrow \bar{a}_{0}$ and $c_{0} \rightarrow \bar{c}_{0}$, the lowest order reaction zone problem can be reduced to the analogous steady problem given by (A.14).

Higher order problems for the reaction zone cannot be so reduced to their steady analogues. The first correction to the solution of (B.6)-(B.8) is discussed 
conveniently in terms of the scaled independent spatial variable $\chi$ :

$$
\begin{aligned}
& \frac{\partial^{2}}{\partial \chi^{2}}\left(\mathscr{F}_{1}-\mathscr{H}_{1}\right)=\frac{d h_{*}}{d t}-\left[\varepsilon(t) \xi_{*}(t)+\frac{d \xi_{*}}{d t}\right] \frac{\partial}{\partial \chi}\left(\mathscr{F}_{0}-\mathscr{H}_{0}\right), \\
& \frac{\partial^{2}}{\partial \chi^{2}}\left(\mathscr{Y}_{1}-\mathscr{H}_{1}\right)=\frac{d h_{*}}{d t}-\left[\varepsilon(t) \xi_{*}(t)+\frac{d \xi_{*}}{d t}\right] \frac{\partial}{\partial \chi}\left(\mathscr{Y}_{0}-\mathscr{H}_{0}\right), \\
& \frac{\partial^{2} \mathscr{H}_{1}}{\partial \chi^{2}}+\frac{d h_{*}}{d t}+\left[\varepsilon(t) \xi_{*}(t)+\frac{d \xi_{*}}{d t}\right] \frac{\partial \mathscr{H}_{0}}{\partial \chi} \\
& =\frac{\partial^{2} \mathscr{H}_{0}}{\partial \chi^{2}}\left\{v_{0} \frac{\mathscr{Y}_{1}}{\mathscr{Y}_{0}}+v_{F} \frac{\mathscr{F}_{1}}{\mathscr{F}_{0}}-\frac{\theta \mathscr{H}_{0}}{\left[h_{*}(t)+\alpha\right]^{2}}\right\} .
\end{aligned}
$$

From (B.11) and (B.12), in view of (4.6),

$$
\left(\begin{array}{l}
\mathscr{F}_{1}-\mathscr{H}_{1} \\
\mathscr{Y}_{1}-\mathscr{H}_{1}
\end{array}\right)=-\left\{\left[\varepsilon(t) \xi_{*}(t)+\frac{d \xi_{*}}{d t}\right] \frac{\chi^{2}}{2}\right\}\left(\begin{array}{l}
\bar{c}_{0} \\
\bar{a}_{0}
\end{array}\right) \text {. }
$$

Appendix C. Consumption of a fuel cylinder under radial compression. For incompressible constant-property axisymmetric flow, if (in dimensional quantities)

$$
w_{z}(r, z, t)=2 \varepsilon_{0}, \quad \text { then } u(r, z, t)=-\varepsilon_{0} r .
$$

Hence,

$$
\left\{\frac{\partial}{\partial t}-\varepsilon_{0} r \frac{\partial}{\partial r}-\frac{D_{\infty}}{r} \frac{\partial}{\partial r}\left(r \frac{\partial}{\partial r}\right)\right\} V(r, t)=0,
$$

where

$$
V(r, t)=\frac{F-\left(Y-Y_{\infty}\right)}{F_{i}+Y_{\infty}} .
$$

Then the consumption of a cylinder of fuel $F$ with initial (stoichiometrically adjusted) mass fraction $F_{i}$, immersed in an unbounded expanse of oxidant $Y$ with initial mass fraction $Y_{\infty}$ (maintained at $r \rightarrow \infty$ ), is described in the thin-flame limit by

$$
\left\{\frac{\partial}{\partial \tau}-4 \bar{\varepsilon} \frac{\partial}{\partial r}-\frac{1}{r} \frac{\partial}{\partial r}\left(r \frac{\partial}{\partial r}\right)\right\} V=0,
$$

where, with subscript denoting partial differentiation,

$$
\begin{aligned}
& V_{r}(0, t)=0, \quad V(r \rightarrow \infty, t)=0, \\
& V(r>1, t=0)=0, \quad V(r<1, t=0)=1 .
\end{aligned}
$$

In (C.4)-(C.6), $\bar{\varepsilon}=\varepsilon_{0} a^{2} / 4 D_{\infty}$, where $a$ is the radius of the cylinder; $\tau=D_{\infty} t / a^{2}$; $r^{\prime}=r / a$, and the prime has been dropped.

$$
\text { If } p=r^{2} \text {, }
$$

$$
V_{\tau}-4\left(p V_{p}\right)_{p}-4 \bar{\varepsilon} p V_{p}=0 \text {. }
$$

Under Laplace transformation in $p$, with $s$ being the transform variable, (C.7) 
becomes, under (C.5),

(C.8)

$$
4(s+\bar{\varepsilon})(s \bar{V})_{s}+\bar{V}_{\tau}=0 .
$$

If one defines $f(s)$ by

$$
-d f=\frac{d s}{4 \bar{\varepsilon}}\left[\frac{1}{s+\bar{\varepsilon}}-\frac{1}{s}\right] \text { so that } f=-\frac{1}{4 \bar{\varepsilon}} \ln \left[\frac{s+\bar{\varepsilon}}{s}\right],
$$

then (C.8) becomes

$$
\bar{V}_{f}+\bar{V}_{\tau}-\frac{4 \bar{\varepsilon} \exp (-4 \bar{\varepsilon} f)}{1-\exp (-4 \bar{\varepsilon} f)} \bar{V}=0
$$

If one now defines

$$
\alpha=(f+\tau) / 2, \quad \beta=(f-\tau) / 2,
$$

so that

$$
\bar{V}_{\alpha}-\frac{4 \bar{\varepsilon} \exp [-4 \bar{\varepsilon}(\alpha+\beta)]}{1-\exp [-4 \bar{\varepsilon}(\alpha+\beta)]} \bar{V}=0
$$

then

(C.13)

$$
\bar{V}=g(\beta)\{1-\exp [-4 \bar{\varepsilon}(\alpha+\beta)]\} .
$$

The initial condition (C.6) implies that

$$
\bar{V}_{(\tau=0)}=\frac{1-\exp (-s)}{s}
$$

and when one notes that

$$
\beta=\frac{f}{2}=\frac{-1}{8 \bar{\varepsilon}} \ln \left[\frac{s+\bar{\varepsilon}}{s}\right]
$$

(C.13) implies

$$
g(\beta)=-\frac{1}{\bar{\varepsilon}}\left\{1-\exp \left[\frac{\bar{\varepsilon}}{1-\exp (-8 \bar{\varepsilon} \beta)}\right]\right\} .
$$

With $\alpha$ and $\beta$ replaced by their equivalents from (C.9) and (C.11), (C.13) then becomes

$$
1-V(p, \tau)=\frac{1}{2 \pi i} \int_{c-i \infty}^{c+i \infty} s^{-1} \exp \left\{s\left[p-(b s+\bar{c})^{-1}\right]\right\} d s
$$

where

$$
b=[\exp (4 \bar{\varepsilon} \tau)-1] / \bar{\varepsilon}, \quad \bar{c}=\exp (4 \bar{\varepsilon} \tau) .
$$

We seek $\tau_{f}$ where

$$
V\left(p \rightarrow 0, \tau=\tau_{f}\right)=\frac{Y_{\infty}}{Y_{\infty}+F_{i}},
$$


i.e., the time at which the thin flame collapses to the origin. But the solution for $p \rightarrow 0$ may be obtained by examining (C.16) for $s \rightarrow \infty$ :

(C.19) $\frac{\exp \left(b^{-1}\right)}{\left[\left(Y_{\infty} / F_{i}\right)+1\right]} \doteq \frac{1}{2 \pi i} \int_{c-i \infty}^{c+i \infty} \frac{\exp \left[s p+\bar{c} / b^{2} s\right]}{s} d s=\lim _{p \rightarrow 0} I_{0}\left(\frac{2 p^{1 / 2}}{b / c^{1 / 2}}\right) \rightarrow 1$.

Hence we obtain

$$
\frac{\exp \left[4 \bar{\varepsilon} \tau_{f}\right]-1}{\bar{\varepsilon}}=\frac{1}{\ln \left[\left(Y_{\infty}+F_{i}\right) / F_{i}\right]} .
$$

Acknowledgment. The authors are very grateful to Dr. J. Eugene Broadwell of TRW Systems, Redondo Beach, California, for advice, criticism and encouragement.

\section{REFERENCES}

[1] G. BROWN AND A. Roshko, The effect of density difference on the turbulent mixing layer, Turbulent Shear Flow, AGARD Conference Proc. 93, Advisory Group for Aerospace Research and Development, Neuilly-Sur-Seine, France, 1972, pp. 23-1 to 23-12.

[2] H. S. Carslaw and J. C. Jaeger, Conduction of Heat in Solids, 2nd ed., Clarendon Press, Oxford, England, 1959.

[3] J. F. ClaRKe, Reaction-broadening in a hydrogen-oxygen diffusion flame, Proc. Roy. Soc. Ser. A, 312 (1969), pp. 65-83.

[4] —_ The diffusion flame as a singular perturbation problem, J. Engrg. Math., 5 (1971), pp. 179-185.

[5] J. F. Clarke AND G. R. Stegen, Some unsteady motions of a diffusion flame sheet, J. Fluid Mech., 34 (1968), pp. 343-358.

[6] F. E. FENDELL, Ignition and extinction in combustion of initially unmixed reactants, Ibid., 21 (1965), pp. 281-303.

[7] - Flame structure in initially unmixed reactants under one-step kinetics, Chem. Engrg. Sci., 22 (1967), pp. 1829-1837.

[8] S. K. Friedlander AND K. H. Keller, The structure of the zone of diffusion controlled reaction, Ibid., 18 (1963), pp. 365-375.

[9] R. M. Fristrom and A. A. Westenderg, Flame Structure, McGraw-Hill, New York, 1965.

[10] D. R. Kassoy AND F. A. Williams, Effects of chemical kinetics on near equilibrium combustion in nonpremixed systems, Phys. Fluids, 11 (1968), pp. 1343-1351.

[11] D. R. Kassoy, M. K. LiU AND F. A. Williams, Comments on "Effects of chemical kinetics on near equilibrium combustion in nonpremixed systems," Ibid., 12 (1969), pp. 265-267.

[12] A. LiÑan, On the structure of laminar diffusion flames, Thesis, Division of Engineering, California Institute of Technology, Pasadena, 1963.

[13] J. R. A. Pearson, Diffusion of one substance into a semi-infinite medium containing another with second-order reaction, Appl. Sci. Res., A11 (1963), pp. 321-340.

[14] D. E. RoSNER, Diffusion theory of reactive chemical releases, Aerochem Research Laboratories Rep. TP-128, Princeton, New Jersey, 1966.

[15] - On liquid droplet combustion at high pressure, AIAA J., 5 (1967), pp. 162-164.

[16] F. A. Williams, Combustion Theory, Addison-Wesley, Reading, Mass., 1965.

[17] - Theory of combustion in laminar flows, Annual Review of Fluid Mechanics, vol. 3, M. Van Dyke, W. G. Vincenti and J. V. Wehausen, eds., Annual Reviews, Palo Alto, California, 1971, pp. 171-188. 